\title{
Fundamentally Different Roles of Neuronal TNF Receptors in CNS Pathology: TNFR1 and IKK $\beta$ Promote Microglial Responses and Tissue Injury in Demyelination, TNFR2 Protects in Excitotoxicity in Mice
}

Irini Papazian

Hellenic Pasteur Institute

Eleni Tsoukala

Hellenic Pasteur Institute

Maria Karamita

Hellenic Pasteur Institute

Athena Boutou

Hellenic Pasteur Institute

Lida lliopoulou

Hellenic Pasteur Institute

Konstantinos Kambas

Hellenic Pasteur Institute

Roman Fischer

Institute of Cell Biology \& Immunology

Roland Kontermann

Institute of Cell Biology \& Immunology, University of Stuttgart

Maria Denis

BSRC Alexander Fleming: Biomedical Sciences Research Center Alexander Fleming

George Kollias

Alexander Fleming Biomedical Sciences Research Centre

Hans Lassmann

Medical University of Vienna Center for Brain Research

Lesley Probert ( $\sim$ lesley.probert@gmail.com )

Hellenic Pasteur Institute https://orcid.org/0000-0002-5931-2880

Research 
Keywords: neuroinflammation, immunotherapy, multiple sclerosis, NF-KB, autophagy, neuroprotection, neurodegeneration, oligodendrocyte death, glutamate excitotoxicity, TNFR2 agonist

Posted Date: January 5th, 2021

DOl: https://doi.org/10.21203/rs.3.rs-136877/v1

License: (c) (1) This work is licensed under a Creative Commons Attribution 4.0 International License. Read Full License 


\section{Abstract}

\section{BACKGROUND}

During inflammatory demyelination TNF receptor 1 (TNFR1) mediates detrimental proinflammatory effects of soluble TNF, whereas TNFR2 mediates beneficial effects of transmembrane TNF through oligodendrocytes, microglia, and possibly other cell types. This model supports use of selective inhibitors of soluble TNF/TNFR1 as antinflammatory drugs for CNS disease. A potential obstacle is the neuroprotective effect of soluble TNF pretreatment described in cultured neurons, but the in vivo relevance is unknown.

\section{METHODS}

To address this question we generated mice with neuron-specific depletion of TNFR1, TNFR2 or IKK $\beta$ and applied experimental models of inflammatory demyelination and acute and preconditioning glutamate excitotoxicity. We also investigated the molecular and cellular requirements of soluble TNF (and therefore TNFR1) neuroprotection by generating astrocyte-neuron co-cultures with different combinations of wildtype and TNF and TNF receptor knockout cells and measuring NMDA excitotoxicity in vitro.

\section{RESULTS}

Neither neuronal TNFR1 nor TNFR2 protected mice during inflammatory demyelination. In fact, both neuronal TNFR1 and neuronal IKK $\beta$ promoted microglial responses and tissue injury, and TNFR1 was further required for oligodendrocyte loss and axonal damage in cuprizone demyelination. In contrast, neuronal TNFR2 increased preconditioning protection in a kainic acid excitotoxicity model in mice, and limited hippocampal neuron death. The neuroprotective effects of neuronal TNFR2 observed in vivo were further investigated in vitro. Here as expected, pretreatment of astrocyte-neuron co-cultures with soluble TNF protected them against NMDA excitotoxicity. However, protection was dependent on astrocyte, not neuronal TNFR1, on astrocyte transmembrane TNF-neuronal TNFR2 interactions, and was reproduced by a TNFR2 agonist.

\section{CONCLUSIONS}

These results demonstrate that neuronal TNF receptors perform fundamentally different roles in CNS pathology in vivo, with neuronal TNFR1 and IKK 3 promoting microglial inflammation and neurotoxicity in demyelination, and neuronal TNFR2 mediating neuroprotection in excitotoxicity. They also reveal that previously-described neuroprotective effects of soluble TNF (and therefore TNFR1) against glutamate excitotoxicity in vitro are indirect, and mediated by astrocyte transmembrane TNF-neuron TNFR2 interactions. These results consolidate the concept that selective inhibition of soluble TNF/TNFR1 with maintenance of TNFR2 function would have anti-inflammatory and neuroprotective properties required for the safe treatment of CNS disease. 


\section{Introduction}

TNF is a multifunctional cytokine system with diverse roles in CNS, ranging from the regulation of physiological functions such as synaptic transmission and plasticity to the induction of inflammation, excitotoxicity and oxidative tissue damage under disease conditions. TNF also has important beneficial functions in demyelinating diseases where it mediates the recruitment and differentiation of oligodendrocyte precursor cells (OPC) and remyelination [1, 2]. The relative contribution of beneficial over deleterious TNF functions to one human demyelinating disease, multiple sclerosis (MS), are clearly illustrated by clinical data, which show that non-selective TNF inhibitors exacerbate disease in MS patients [3], and induce de novo demyelination in patients treated for other diseases [4, 5]. Also, TNFRSF1A is a risk gene for MS where it encodes a soluble form of TNF receptor 1 that can block TNF [6, 7]. These data suggest that beneficial TNF functions in remyelination and suppression of inflammation outweigh deleterious ones and are important for CNS resistance to demyelination.

Understanding the mechanisms of TNF effect in the CNS is important for the refinement of TNF inhibitors as safe therapeutics for various inflammatory diseases including MS. The TNF system involves two ligands and two receptors [8]. Soluble TNF (solTNF) signals mainly through TNFR1 to mediate deleterious TNF effects [9, 10], including an inhibitory effect on remyelination [11]. Transmembrane TNF (tmTNF) signals mainly through TNFR2 and mediates beneficial effects, such as remyelination [12]. This information provides an important mechanistic framework upon which to base the design of novel selective TNF inhibitors for the treatment of CNS inflammatory diseases [13].

One potential obstacle for selective inhibition of solTNF/TNFR1 signaling as an anti-inflammatory therapy in CNS disease is its role in neuroprotection. Pretreatment of cultured neurons with solTNF (TNF preconditioning) protects them against a wide range of metabolic, excitotoxic and oxidative death stimuli and promotes maintenance of calcium homeostasis $[14,15]$. Whether these effects are exerted directly by TNF signaling in neurons or indirectly by small numbers of glial cells in the cultures, and are relevant in vivo remains unknown. Studies in conventional knockout mice showed worsening of CNS pathology in experimental stroke in TNFR1 knockout (TNFR1KO) mice [16], and in experimental autoimmune encephalomyelitis (EAE) and retinal ischemia in TNFR2KO mice [17-19], but the mechanisms underlying these protective effects are poorly understood. The availability of mutant mice carrying conditional TNFR1 and TNFR2 alleles now allow the cell specificity of TNF effects to be elucidated. Elegant studies recently showed that depletion of TNFR2 selectively in microglia [20] or oligodendroglia [21] led to early onset or enhanced disease respectively, in EAE, showing that glial cell-specific beneficial functions of TNFR2 are sufficient to protect the CNS during inflammatory demyelination.

To investigate the relevance of neuronal TNFR for CNS disease, especially their possible roles in neuroprotection, we generated mice with neuron-specific depletion of TNFR1, TNFR2 or IKK $\beta$, a main downstream mediator of TNFR signaling [22], and used them with models of inflammatory demyelination and glutamate excitotoxity. We also used astrocyte-neuron co-cultures with different combinations of wild type, TNFKO, tmTNFKI and TNFRKO cells and glutamate excitotoxicity models in vitro, to verify and 
further define the molecular and cellular interactions underlying TNFR-mediated neuroprotection. Here we show that neuronal TNFR1 and TNFR2 have distinct inflammatory/neurotoxic and neuroprotective effects, in mouse models of CNS demyelination and excitotoxicity, respectively. Specifically, neuronal TNFR1 and IKK $\beta$ both advance the onset of inflammatory demyelination, TNFR1 is further required for axon damage and OLG loss during cuprizone demyelination, and neuronal TNFR2 increases protection against glutamate excitotoxicity in vitro and in vivo. These results consolidate the concept that selective inhibition of solTNF/TNFR1 with maintenance of TNFR2 function would have anti-inflammatory and neuroprotective properties required for the safe treatment of CNS disease.

\section{Materials And Methods}

\section{Animals}

For in vitro experiments, male and female E14.5 embryos and P0 mice were used for the isolation of cortical neurons and cortical astrocytes, respectively, from wildtype (WT) C57BL/6 (B6), Tnf-/-(TNFKO; [23]), tmTnf $\Delta / \Delta$ (tmTNFKI; [24], a gift of B. Ryffel, University of Orleans and CNRS, INEM, Molecular Immunology, Orleans, France), Tnfrsf1a-/-(TNFR1KO; [25], a gift of H. Bluethmann, Hoffmann-La Roche Ltd, Basel, Switzerland), and Tnfrsf1b-/-(TNFR2KO; [26]) mice. For in vivo experiments, mice with conditional inactivation of TNFR1, TNFR2 or IKK $\beta$, the main activating kinase for NF-KB in the canonical pathway and main downstream mediator of TNFR1 signaling $[8,22]$, selectively in CNS neurons were generated by Cre-LoxP recombination technology. The generation and initial characterization of TNFR1 conditional knockout mice has been described previously [27]. Briefly, mice in which exons 2-5 of the Tnfrsf $1 a$ allele are flanked by LoxP sites were generated using standard gene-targeting techniques (named here TNFR1ff mice). The generation and initial characterization of TNFR2 conditional knockout mice has been described previously [28]. Briefly, mice in which exon 2 of the Tnfrsf $1 b$ allele is flanked by LoxP sites were generated using standard gene-targeting techniques (named here TNFR2ff mice). Mice in which exon 3 of the lkbkb gene is flanked by LoxP sites (IKKßff mice, a gift of M. Karin, Department of Pharmacology, University of California at San Diego, California, USA), have been described previously [29]. Mice with a selective depletion of TNFR1, TNFR2 or IKK 3 in excitatory CNS neurons (nTNFR1KO, nTNFR2KO, nIKKßKO) were generated by crossing TNFR1ff, TNFR2ff, or IKKßff mice with mice that express a neuronal calmodulin-kinase lla promoter-driven Cre recombinase (CamkllCre mice; [30]). TNFR1ff, TNFR2ff, and IKKßff littermate mice were used as respective controls in all in vivo experiments.

DNA PCR analysis for the detection of recombination (deletion) events in different tissues of nTNFR1KO mice was performed using 2 independent PCR reactions. Primers sense 5'-CAA GTG CTT GGG GTT CAG GG-3' and antisense 5'-CGT CCT GGA GAA AGG GAA AG-3' (ThermoFisher Scientific) were used for the detection of wildtype Tnfrsf1a (134bp band) and floxed TNFR1ff alleles (195bp band). Primers sense 5'CCT-GCA-GAC-ACA-CGG-GGA-AA -3' and antisense 5'- TGA-ACT-CAG-GTT-GCC-AGA-CG-3' (ThermoFisher Scientific) were used for the detection of recombined (deleted, "defloxed") allele (300bp band). DNA PCR analysis for the detection of recombination (deletion) events in tissues of nTNFR2KO mice was performed using a combination of 3 primers in one PCR reaction; sense 5'-CAC ATG TAT GTA CAC CTG 
TGT G-3', antisense 5'-CTC TCC TGG GCC TAA TGT AG-3', and antisense 5'-ATT GTC TAC TCA GCA CTG GG-3' (ThermoFisher Scientific) for the detection of wildtype Tnfrsf1b allele (151 bp band), floxed TNFR2ff (200 bp band), and recombined (deleted, "defloxed") alleles (321 bp band). Detection of Cre recombinase was performed using primers, sense 5'-ATT ACC GGT CGA TGC AAC GAG T-3' and antisense 5'-CAG GTA TCT CTG ACC AGA GTC A-3' (800bp band). All mice were backcrossed and maintained on the B6 genetic background. Animals were maintained under specific pathogen-free conditions at the animal facilities of the Hellenic Pasteur Institute and all experimental procedures were approved by national authorities and conformed to ARRIVE guidelines and EU Directive 2010/63/EU for animal experiments.

\section{EAE}

EAE was induced in 10-12-week-old female nTNFR1KO and nTNFR2KO mice, and their respective TNFR1ff and TNFR2ff littermate controls as previously described [31]. EAE was induced by s.c. tail base injection of $37 \mu \mathrm{g}$ of rat myelin oligodendrocyte glycoprotein 35-55 peptide (MOG) dissolved in $100 \mu$ l of saline and emulsified in $100 \mu$ l CFA (Sigma-Aldrich) supplemented with additional $400 \mu \mathrm{g}$ of H37Ra Mycobacterium tuberculosis (Sigma-Aldrich) (MOG/CFA). Mice also received an i.p. injection of $200 \mathrm{ng}$ of Bordetella pertussis toxin (PTx) (Sigma-Aldrich) on days 0 and 2. Mice were assessed daily for clinical signs as previously described [31]. Briefly, mice were examined using an EAE scoring scale as follows: grade 0, no clinical symptom; grade 1, limp tail; grade 2, hindlimb weakness; grade 3, hindlimb paralysis; grade 4, forelimb and hindlimb paralysis; grade 5, moribund or dead. Animals with score 4 or above were euthanized. Mice were allowed free access to food and water throughout the experiment.

\section{CPZ-induced demyelination and remyelination}

CPZ induced demyelination was induced in 8-10-week-old male nTNFR1KO, nTNFR2KO, and nIKK $\beta K O$ mice and their respective TNFR1ff, TNFR2ff and IKKßff littermate controls. Mice were fed ad libitum with 0.2\% w/w CPZ (Sigma-Aldrich; C9012) in powdered standard mouse chow using feeder devices (Analab, Tecniplast, Italy) for 6 weeks and then returned to normal diet, as described [32]. Consistent with this model, hallmark clinical features of disease were measured in the midline corpus callosum of CPZtreated mice at the end of the second week of CPZ feeding (CPZ2) (microglial response), CPZ3 (microgliosis, axonal damage, and demyelination by LFB staining), CPZ5 (maximal demyelination by immunohistochemical staining), and several time-points after removal of CPZ from the diet at week 6 of feeding, including one week after cessation of CPZ feeding (CPZ6+1) (remyelination), CPZ6+2 and CPZ6+4 (both almost complete remyelination) [11].

\section{Acute and preconditioning KA excitotoxicity models in mice}

In an acute KA seizure model, 2-6-month-old male nTNFR1KO and nTNFR2KO mice, and their respective TNFR1ff or TNFR2ff littermate controls, were injected i.p with 20 or $24 \mathrm{mg} / \mathrm{kg} \mathrm{KA}$ according to manufacturer's instructions (Tocris Bioscience). In a modified preconditioning KA protocol based on a previously described model [33], mice were injected i.p. with $15 \mathrm{mg} / \mathrm{kg} \mathrm{KA}$ and subsequently after $24 \mathrm{~h}$ i.p. with $20 \mathrm{mg} / \mathrm{kg} \mathrm{KA}$. Seizures were scored every 5 min for $90 \mathrm{~min}$, using a clinical seizure scale based on 
previously described methods [34, 35]. Briefly, mice were scored as follows: 0 , no behavioral response; 1 , immobility; 2 , one or more of the following: head bobbing, whiskers movement, hunching, outstretched forelimbs; 3 , rearing and one-sided forelimb spasms; 4 , repeated rearing and two-sided forelimb spasms; 5 , one or more of the following: convulsions with sideways leaning, jumping and wild running followed by convulsions, death. Animals with score 5 were euthanized.

\section{RNA isolation and quantitative RT-PCR}

Brain was removed and spinal cord was flushed from the vertebral column after transcardial perfusion with ice-cold PBS at sacrifice by carbon dioxide inhalation. Total RNA was extracted from spinal cords and brains using TRIzol reagent (Thermo Fisher Scientific), according to the manufacturer's instructions. RNA purity and quantification were assessed using NanoDrop 2000 spectrophotometer data. RNA was used for one-step quantitative RT-PCR using a QuantiFastTM SYBR® green RT-PCR kit (Qiagen Inc.) and QuantiTect Primer Assays for Ccl2 (Mm_Ccl2_1_SG), Cxcl16 (Mm_Cxcl16_1_SG), H2Ab1 (Mm_H2-Ab1_1_SG), Mbp (Mm_Mbp_1_SG), Olig2 (Mm_Olig2_1_SG), Snap25(Mm_Snap25_2_SG), Tnf (Mm_Tnf_1_SG), and Gusb (Mm_Gusb_1_SG) (Qiagen Inc). All reactions were performed in duplicate using the LightCycler system (Roche, Mannheim, Germany). At the end of each PCR run, melting curve analysis was performed to verify the integrity and homogeneity of PCR products. Results were analyzed using LightCycler software version 3.5 (Roche Diagnostics). Normalization was done using Gusb as a reference gene. Two methods were used for measuring RNA levels, with the same results. For spinal cord samples from EAE experiments, gene expression levels were calculated using already created standard curves for each gene as previously described [36]. These standard curves were created by plotting threshold cycle values vs the logarithm of serial diluted RNA concentrations. Least-squares methods were used for the determination of $A$ and $B$ values in the equation threshold cycle $=A^{*} \log (C R N A)+B$. The coefficient of determination (R2) was greater than 0.99. A second method was used for CPZ brain samples, except for Snap25, which was calculated as above. The difference between threshold cycle values of the target and reference gene $(\triangle \mathrm{CT}=\mathrm{Ct}$ target gene $-\mathrm{Ct}$ reference gene) in each sample was calculated. The relative gene expression was then estimated according to the $\Delta \Delta$ threshold cycle method in which $\Delta \Delta C T=(\Delta C T$ of a target sample) $-(\Delta C T$ of the average of reference/ naïve samples). The final relative gene expression was expressed as $2^{-\Delta \Delta C T}$ value.

\section{Histology and immunohistochemistry}

Mice were transcardially perfused with ice-cold $4 \%$ paraformaldehyde in PBS at sacrifice by carbon dioxide inhalation. For analysis of CPZ-induced pathology, brains were removed, post-fixed in the same fixative overnight at $4^{\circ} \mathrm{C}$ and embedded in paraffin. For histology and immunohistochemistry (IHC), serial coronal paraffin sections $(5 \mu \mathrm{M})$ were cut through the corpus callosum corresponding to Sidman sections 295-305, and comparative analyses were made in the midline corpus callosum. Sections were stained with Klüver-Barrera Luxol fast blue (LFB) for demyelination [11]. IHC was performed as described [11] using the primary antibodies mouse anti-2', 3'-cyclic nucleotide 3'-phosphodiesterase (CNPase) mAb (1/700; clone SMI-91; Covance), mouse anti-amyloid precursor protein APP (1/350, Covance), rabbit anti- 
ionized calcium-binding adaptor molecule 1 (Iba1) (1/400; Wako chemicals; 019-19741), mouse antineurofilament H phosphorylated (SMI 31) (1/1000; clone SMI-31; Covance), rabbit anti-glial fibrillary acidic protein (GFAP) (1/300; Dako; Z0334), anti-apoptosis inducing factor (AIF), anti-CD3 (145-2C11; BD Pharmingen); followed by biotinylated secondary Abs, horseradish peroxidase-labeled avidin-biotin complex, and visualized using 3'3'-diaminobenzidine (DAB) (all from Vector Laboratories). Detailed analysis of pathology in our MOG-induced EAE model has previously been described $[10,30]$. Immunofluorescence staining for autophagy detection was performed as previously described [37]. Briefly, paraffin sections were immunolabelled using a polyclonal rabbit anti-mouse LC3B antibody (1:200; Sigma-Aldrich, L7543), followed by goat anti-rabbit Alexa Fluor 647 (1:1000; ThermoFisher Scientific). Immunofluorescence was observed and scanned with a $60 x$ objective using a Leica TCS-SP8 Confocal Microscope.

\section{Histology quantification}

Tissue sections were viewed with an Olympus BX-50 microscope and images captured with an Olympus DP71 microscope digital camera using cell^A imaging software (Soft Imaging System $\mathrm{GmbH}$ ). Quantitative histopathological analysis was performed in midline corpus callosum, unless otherwise stated using Image J software. Demyelination was measured as loss of LFB staining in coronal sections of whole corpus callosum using a semi quantitative method, as described [11, 12], and loss of CNPase immunostaining in the midline corpus callosum by densitometry. Axonal damage was measured as the numbers of positive APP-stained spheroids per $\mathrm{mm}^{2}$ tissue, and loss of SMI 31 immunostaining by densitometry. Microglia and astrocyte responses were measured as the area covered by Iba1- and GFAPimmunoreactivity, respectively. AlF levels were measured as the area covered by AIF-immunoreactivity, and $\mathrm{CD}^{+} \mathrm{T}$ lymphocytes were counted as cells $/ \mathrm{mm}^{2}$. Autophagy induction was measured as the numbers of LC3 puncta/LC3-immunoreactive cell in (lateral) corpus callosum area as previously described [38], using a macro developed in Fiji software. Fifty LC3-immunoreactive cells were analyzed for each condition (CPZO and CPZ5 control and nTNFR1KO mice).

\section{Cortical astrocyte-neuron co-cultures}

Astrocyte cultures were prepared from cortical tissues of male and female postnatal mice P0 ( $1 \mathrm{~d}$ after birth), based on a previously described method [39] (Supplementary Figure 4). Briefly, cells were grown in $25 \mathrm{~cm}^{2}$ flasks, pre-coated with poly-D-lysine $(0.1 \mathrm{mg} / \mathrm{ml})$ and laminin $(20 \mathrm{mg} / \mathrm{ml})$ (Sigma-Aldrich; P6407), in high glucose medium supplemented with $10 \%$ horse serum, $10 \%$ fetal bovine serum, $0.5 \mathrm{mM}$ glutamine and $50 \mathrm{U} / \mathrm{ml}$ penicillin-streptomycin. Cultures were grown to confluency by around 7-10 day in vitro (DIV7-10), harvested and re-seeded on poly-D-lysine/laminin-coated glass coverslips in 48-well plates (40.000 cells/well). They were used for co-culture with neurons on DIV5.

Cortical neurons were isolated from male and female E14.5 embryos, as previously described [40]. To prepare astrocyte-neuron co-cultures (NA), neurons were seeded onto the coverslips coated with confluent DIV5 astrocytes in 48-well plates (180.000 neurons/well) in Neurobasal medium supplemented with $2 \%$ 
$\beta-27,0.5 \mathrm{mM}$ glutamine and $50 \mathrm{U} / \mathrm{ml}$ penicillin-streptomycin. After $2 \mathrm{~d}$ of co-culture (NA-DIV2), proliferation of non-neuronal cells was halted by addition of $10 \mu \mathrm{M}$ Ara-C for $2 \mathrm{~d}$. At NA-DIV7, recombinant mouse (R\&D systems; 410-MT-010), or human (R\&D systems; $210-T A-010)$ TNF (100 ng/ml) was added for 24 hours. Excitotoxic death was induced in NA-DIV8 co-cultures by exposure to $50 \mu \mathrm{M}$ NMDA (Sigma-Aldrich; M3262) supplemented with $10 \mu \mathrm{M}$ glycine for $22 \mathrm{~h}$, followed by fixation with $4 \%$ paraformaldehyde in PBS on NA-DIV9. The cells were immunolabelled with mouse anti-NeuN (1/300; Chemicon), rabbit anti- GFAP (1/400; DAKO) followed by goat anti-mouse Alexa Fluor 488 (1/1000; Molecular Probes) or anti-rabbit Alexa Fluor 568 (1/500; Molecular Probes) antibodies and counterstained with Hoechst 33342. Immunostaining with rabbit anti-lba1 (1/400; Wako chemicals; 01919741), showed approximately $5 \%$ of cells in co-cultures were microglia. Excitotoxic death was measured by the percentage of Hoechst 33342-stained pyknotic nuclei in NeuN-positive neurons. Pyknotic nuclei are characteristic of neuronal necrosis induced by acute excitotoxicity in vivo and in vitro [41]. The NMDA receptor antagonist MK801 $(10 \mu \mathrm{M})$ was added to culture medium of control cells together with NMDA for $22 \mathrm{~h}$.

\section{Statistics}

Statistical analyses were performed using Graphpad Prism 7. Data are presented as mean \pm SEM. Student's $t$ test, one-way ANOVA or Mann-Whitney was performed for pairwise comparisons between neuron viabilities as measured by Hoechst staining, relative mRNA levels and EAE or KA scores between animals at each point in the different groups. Two-way ANOVA (Bonferroni test) and Student's $t$ test were used for longitudinal analysis of CPZ experiments or pairwise comparisons at specific time-points, respectively. Values of $p \leq 0.05$ were considered statistically significant.

\section{Results}

\section{Neuronal TNFR1 promotes neuroinflammation and the onset of EAE}

With the aim of interpreting the opposing neurotoxic and neuroprotective effects of the TNF cytokine system described in the literature, we studied the effects of neuronal TNFR1 and TNFR2 using two CNS demyelination models, experimental autoimmune encephalomyelitis (EAE) and cuprizone-induced demyelination (CPZ). We crossed TNFR1ff and TNFR2ff mice with CamkII-Cre mice to achieve TNFR1 or TNFR2 depletion selectively in glutamatergic excitatory CNS neurons. Neuron-specific TNFR1KO (nTNFR1KO) and TNFR2KO (nTNFR2KO) mice were born at the expected Mendelian ratio, were viable, fertile and didn't display any spontaneous phenotypes. Efficiency and specificity of TNFR1 and TNFR2 deletion in brain and spinal cord tissues was verified by DNA analysis which showed recombination specifically in brain and spinal tissues, and not any other tissue tested (Supplementary Figure 1).

nTNFR1KO, nTNFR2KO, and their respective TNFR1ff and TNFR2ff littermate controls were immunized with MOG to induce EAE, a disease driven by myelin-reactive $T$ cells and other infiltrating immune effector cells. In our MOG-EAE model in B6 mice pathology is characterized by severe infiltration of the meninges 
and spinal cord parenchyma by immune cells (including CD $4^{+}$and $\mathrm{CD} 8^{+} \mathrm{T}$ cells, B cells, NK $1.1^{+}$cells and myeloid cells), microglia and astrocyte activation, demyelination and axonal damage in the white matter at the peak of disease. Pathology partially resolves during the chronic phase of disease [30].

nTNFR1KO, nTNFR2KO and control mice all showed full susceptibility to EAE (Figure 1). nTNFR1KO mice showed a small but significant delay in disease onset compared to TNFR1ff controls in 2 of 3 experiments, but thereafter the two groups of mice showed no differences in clinical disease progression up to the last time point studied (day 70) (Figure 1A). nTNFR2KO mice developed EAE with equal onset and clinical course as TNFR2ff littermate controls up to the last time point studied (day 38) (Figure 1C). The results suggest that neuronal TNFR1 plays a small disease-advancing role at the onset of EAE; otherwise, neuronal TNFR1 and TNFR2 do not have significant effects on the clinical course of EAE.

To investigate how neuronal TNFR1 advances EAE onset, RNA was isolated from the spinal cord of nTNFR1KO and TNFR1 ff mice at disease peak in the control group and analyzed for the expression of early-disease marker genes [36]. nTNFR1KO and TNFR1ff spinal cord showed equally marked reduction in expression of myelin $(\mathrm{Mbp})$ and neuronal (Snap25) gene markers compared to corresponding naïve mice confirming the onset of EAE (Figure 1B). However, while TNFR1ff spinal cord showed significantly increased expression of the inflammatory gene marker (H2-Ab1) at disease peak compared to naïve, nTNFR1KO showed no increase (Figure 1B). These results suggest that neuronal TNFR1 promotes an acute neuroinflammatory response in spinal cord tissue involving microglia and astrocytes, and thereby the onset of clinical symptoms in EAE, while neuronal TNFR2 has no obvious effect on disease development.

\section{Neuronal TNFR1 advances the onset of microglia responses and demyelination, and is necessary for OLG loss and axon damage in cuprizone demyelination}

We next used nTNFR1KO, nTNFR2KO, and control mice in an acute toxicity demyelination model induced by 6-weeks of dietary CPZ [32]. CPZ is a copper chelator that causes mitochondrial dysfunction and oxidative stress, both of which are key features of progressive MS [42]. In B6 mice CPZ induces acute responses of microglia and astrocytes, massive death of mature OLG between weeks 2-5 of CPZ feeding that is thought to be triggered by immune mechanisms [43], and predictable primary demyelination in the midline corpus callosum, followed by almost complete remyelination by 2 weeks after removal of CPZ from the diet $[11,32]$.

CPZ-induced pathology in the corpus callosum of nTNFR1KO mice was overall less severe than in TNFR1ff controls. nTNFR1KO mice showed less demyelination at CPZ3 by LFB staining of myelin (Figure 2Ai\&ii), a time point where myelin detected by the more sensitive CNPase immunostaining method appeared still well preserved in both strains. nTNFR1KO mice showed less OLG and myelin loss at CPZ5 by CNPase immunostaining, compared to TNFR1ff controls (Figure 2Bi\&ii). nTNFR1KO mice also showed a reduced microglia response at $\mathrm{CPZ3}$ by Iba1 immunostaining (Figure 2Ci\&ii), and no measurable axon damage at any time point by numbers of axonal APP spheroids, compared to controls (Figure 2Di\&ii). 
Both strains showed full resolution of pathology by 2 weeks after cessation of CPZ feeding (CPZ6+2) indicating that remyelination proceeds normally. These results indicate that the combination of solTNF/TNFR1 signaling and CPZ induces stress in neurons that results in the exacerbation of CPZinduced pathology.

Considering that both TNF signaling and oxidative stress are strong inducers of autophagy [44, 45], and that autophagy in microglia is responsible for the degradation and clearance of myelin debris in vitro [46], we also investigated the effect of neuronal TNFR1 on autophagy in CPZ demyelination. We measured autophagy levels in the brain sections from naïve and CPZ-fed nTNFR1KO and control mice by immunostaining for LC3, a key component of autophagosomes. Autophagic induction was significantly up-regulated in cells located in the corpus callosum (glial cells) of CPZ5 control mice compared to naïve CPZO controls, measured by the numbers of LC3-immunoreactive puncta per LC3-positive cell (Figure 3). On the other hand, levels of autophagic induction were similarly low in CPZ5 and naïve CPZO nTNFR1KO mice, as in naïve control mice, showing an absence of up-regulation. These results suggest that neuronal TNFR1 is necessary for stimulating autophagy in glial cells, probably in myelin-phagocytosing microglia and reflecting the increased levels of demyelination and need for clearance of myelin debris in the control animals.

nTNFR2KO and TNFR2ff mice showed no differences in CPZ-induced pathology, measured by LFB staining, or CNPase, Iba1, and APP immunostaining (Supplementary Figure 2A-D) and both strains showed full resolution of pathology by 2 weeks after cessation of CPZ feeding (CPZ6+2).

These results show that as in EAE, neuronal TNFR1 promotes neuroinflammation and demyelination in the CPZ model. Notably, neuronal TNFR1 contributes to acute microglia responses and is required for damage of both axons and OLG in this model. The effect of neuronal TNFR1 on axon damage might be direct by increasing the oxidative stress initiated by CPZ in neurons themselves, and indirect in both neurons and OLG by increasing microglia responses and the production of pro-inflammatory cytokines and chemokines. As in EAE, neuronal TNFR2 has no effect on disease development in the CPZ demyelination model.

\section{Neuronal IKKßadvances neuroinflammation and the onset of demyelination in cuprizone demyelination}

The pro-inflammatory effects of TNFR1 are dominantly mediated through activation of the transcription factor NF-KB, which in turn induces expression of genes encoding cytokines, chemokines and antiapoptosis molecules [22]. Under disease conditions it is induced and is a critical mediator of inflammation. To examine the link between neuronal TNFR1 and NF-KB signaling in CNS demyelinating disease, we crossed mice carrying a conditional floxed allele for IKK $\beta$ (IKK $\beta f f$ mice), the main NF-KB activating kinase in the canonical NF-KB pathway [22] with CamkII-Cre mice, to generate nIKK $\beta K O$ mice [30]. We previously showed that nIKKßKO mice have a small but significant delay in EAE onset similar to that described here for nTNFR1KO mice, but unlike nTNFR1KO mice nIKK $\beta K O$ mice subsequently develop a severe non-remitting disease, indicative of neuroprotective effects of neuronal IKK $\beta$ during chronic disease [30]. 
Here we induced CPZ demyelination in nIKKßKO and IKK $\beta f f$ mice. At the onset of pathology at CPZ3, nIKKßKO mice showed reduced demyelination by LFB staining of myelin (Figure 4Ai\&ii), axon damage by numbers of APP-immunoreactive spheroids in axons (Figure 4Di\&ii), and neuroinflammation by lba1 immunostaining of microglia (Figure 5Ai\&ii) and GFAP immunostaining of astrocytes (Figure 5Di\&ii), compared to IKK $\beta f f$ controls, effects that resemble those in nTNFR1KO mice. Unlike nTNFR1KO mice, nIKK $\beta K O$ mice subsequently developed full pathology with loss of myelin and OLG measured at CPZ5 by CNPase immunostaining (Figure 4B). This was further shown by immunostaining for apoptosis-inducing factor (AIF), a mitochondrial oxidoreductase that contributes to apoptosis and has been implicated in CPZ-induced OLG death [43]. AlF-immunoreactivity was increased in the corpus callosum of both IKK $\beta f f$ and nIKK $\beta K O$ mice at CPZ5, most probably in oligodendrocytes which are cells that undergo apoptosis in this model (Figure 4Ci\&ii).

Analysis of whole brain RNA levels for disease marker genes, first for myelin, showed that naïve (CPZ0) nIKKßKO mice express higher levels of $M b p$ in brain compared to IKK $\beta f f$ mice (Supplementary Figure 3A). Expression of $M b p$ and Olig2 dropped during CPZ feeding and increased again in both nIKK $\beta K O$ and IKK $\mathrm{ff}$ mice after CPZ removal, showing myelin recovery independently of neuronal IKK $\beta$ (Supplementary Figure 3A, B). Expression of the neuron-specific gene Snap25, which encodes a protein essential for synaptic function, sharply dropped during CPZ feeding and increased again in both groups after CPZ removal. Interestingly, the rebound increase of Snap25 expression in nIKK $\beta K O$ mice was consistently much higher than in controls (Figure 4E), suggesting that neuronal NF-KB might inhibit recovery of neuronal functions following CPZ toxicity. Expression of inflammatory markers Tnf, CCl2 and CxC/16 in control IKKßff mice showed increases at two distinct time points, first at CPZ2 at the initiation of microglia responses ( $T n f, C c / 2, C x C / 16)$, and again at $C P Z 5$ at the peak of demyelination/initiation of remyelination ( $C c / 2, C x c / 16)$, or $C P Z 6+0,5$ during remyelination (Tnf) (Figure 5B, C, E). Neither Tnf nor Cc/2 showed initial increase in expression at CPZ2 in nIKK $3 K O$ mice, correlating with the delay in glial cell activation observed in these mice at CPZ3 (Figure 5B, C). Also, levels of $C c / 2$ and $C x c / 16$ were significantly lower in nIKK $3 K O$ mice at the peak of disease at CPZ5 (Figure 5C, E). These results support the pathological findings that neuronal IKK $\beta$ promotes neuroinflammation induced by CPZ feeding.

T cells, and specifically IL-17-producing T cells, are reported to participate in CPZ demyelination [47], so we investigated $T$ cell infiltration by counting numbers of CD3-immunoreactive cells in the corpus callosum. Infiltrating CD3-immunoreactive T cells were localized in the corpus callosum of control IKK $\beta f f$ mice at $\mathrm{CPZ5}$, and infiltration was resolved after $\mathrm{CPZ}$ withdrawal at $\mathrm{CPZ6+1}$ and $\mathrm{CPZ6}+4$ (Supplementary Figure 3Ci\&ii). Infiltrating CD3-immunoreactive T cells were also localized in the corpus callosum of nIKK $\beta K O$ mice, but unlike in control IKK $\beta f f$ mice, continued to accumulate after CPZ withdrawal at $\mathrm{CPZ6}+1$, showing late resolution at CPZ6+4 (Supplementary Figure 3Ci\&ii).

These data show that neuronal NF-KB activity, like neuronal TNFR1, plays a role in initiating neuroinflammation (microglia and astrocytes), demyelination and axon damage in response to dietary $\mathrm{CPZ}$, and might have additional, different roles to TNFR1 during disease resolution and recovery. 
Neuronal TNFR2 increases preconditioning protection against seizures and the survival of hippocampal neurons in kainic acid-induced excitotoxicity

Evidence for neuroprotective properties of TNF stems mainly from in vitro studies in which solTNF pretreatment of enriched neuron cultures (TNF preconditioning) protects them against a wide range of metabolic, excitotoxic and oxidative death stimuli and promotes maintenance of calcium homeostasis $[14,15]$. To investigate the role of neuronal TNFR1 or TNFR2 in glutamate excitotoxicity in vivo, we first used an acute kainic acid (KA) excitotoxicity model. KA is a non-degradable glutamate analogue that induces epileptic seizures and death of CA3/CA2 hippocampal neurons in susceptible mouse strains. B6 mice are known to exhibit high seizure scores but no excitotoxic neuron death [34]. Also, low doses of KA are known to protect mice against seizures induced by subsequent higher doses of KA [33], allowing us also to examine the effect of neuronal TNFR in preconditioning protection against glutamate excitotoxicity in vivo.

Acute systemic (i.p.) administration of KA (20 or $24 \mathrm{mg} / \mathrm{kg}$ ) consistently induced seizures in groups of nTNFR1KO, nTNFR2KO and control mice without high mortality. No differences in seizure activity between nTNFR1KO (Figure 6A, grey circles) and TNFR1ff controls (Figure 6A, black circles) or nTNFR2KO (Figure 5B, grey circles) and TNFR2ff controls (Figure 6B, black circles) were observed in this acute model during the 90 minutes of monitoring, although a trend for less severe scores was noted in nTNFR2KO mice compared to TNFR2ff controls (Figure 6B).

We next used a KA preconditioning protocol, adapted from one previously described [33]. In TNFR2ff controls low dosage $K A$ ( $15 \mathrm{mg} / \mathrm{kg}$, i.p.) significantly reduced severity of seizures induced by subsequent high dosage KA (20 mg/kg, i.p.) (Figure 6B, black squares) compared to high dosage KA alone (Figure 6B, black circles), an effect consistent with KA preconditioning protection. In nTNFR2KO mice however, the preconditioning protection effect of low dosage KA on seizures induced by subsequent high dosage KA (Figure 6B, grey squares) compared to high dosage KA alone (Figure 6B, grey circles) was reduced and non-significant. The loss of KA preconditioning protection in nTNFR2KO mice appeared to be a combined effect of reduced acute seizure intensity and reduced preconditioning protection, but these results provide the first indication that neuronal TNFR2 is necessary for effective preconditioning neuroprotection induced by low-dose KA during glutamate excitotoxicity in vivo. A direct protective role for neuronal TNFR2 was further supported by the presence of frequent pyknotic neurons stained by cresyl violet (Nissl), a feature of neuron death, localized in patches in both CA2 and CA3 regions of the hippocampus of nTNFR2KO, but not TNFR2ff mice, 5 days after KA seizures with and without preconditioning (Figure $6 C)$.

\section{Astrocyte TNFR1 and tmTNF via neuronal TNFR2 are necessary for, and a TNFR2 agonist reproduces, TNF preconditioning protection of cortical neurons against NMDA excitotoxicity in vitro.}

To reconcile previously reported neuroprotective effects of solTNF (and therefore TNFR1) against glutamate excitotoxicity in vitro $[14,15]$, with the in vivo findings of TNFR2 neuroprotection here, we modeled different cellular TNF/TNFR interactions in vitro using astrocyte-neuron co-cultures and 
measured glutamate excitotoxicity induced by NMDA in neurons. Mouse cortical neurons and astrocytes were isolated from WT mice, or mice deficient in TNF (TNFKO), soluble TNF (tmTNFKI), TNFR1 (TNFR1KO) or TNFR2 (TNFR2KO), and different combinations of these cells were used for co-cultures.

In this system, exposure of WT astrocyte-neuron co-cultures to NMDA (50 $\mu \mathrm{M})$ supplemented with glycine $(10 \mu \mathrm{M})$ for $24 \mathrm{~h}$ induced neuron death, measured by increased percentage of NeuN-positive neurons with pyknotic nuclei stained by Hoechst (Supplementary Figure 4). Astrocyte viability was not affected by NMDA (data not shown), and neuron death was strongly inhibited by the non-competitive NMDA antagonist MK801 (Supplementary Figure 4). Consistent with previous studies [10,48], pretreatment of WT B6 neuron-astrocyte co-cultures with soluble recombinant hTNF or mTNF $(100 \mathrm{ng} / \mathrm{ml})$ for $24 \mathrm{~h}$ prior to NMDA challenge, consistently and equally protected neurons by $17-20 \%$ against NMDA death, and hTNF was used for all experiments described below (Supplementary Figure 4; Figure 6).

Neuroprotection by hTNF was absent when WT neurons were cultured with TNFKO astrocytes but maintained in WT neurons cultured with tmTNFKI astrocytes, and in TNFKO, tmTNFKI, as well as TNFR1KO neurons cultured with WT astrocytes (Figure 7A). Importantly, neuroprotection was reduced when TNFR2KO neurons were cultured with WT astrocytes (Figure 7A). These results suggest that preconditioning protection of cortical neurons induced by exogenous soluble hTNF is mainly dependent on astrocyte tmTNF-neuronal TNFR2 interactions. Under these conditions, neuronal TNFR1 also has a very small neuroprotective effect, detectable in WT astrocyte- TNFR2KO neuron co-cultures (Figure 7A).

To understand how soluble hTNF triggers this mechanism, we performed experiments using TNFR1KO neurons and astrocytes. hTNF signals efficiently through mouse TNFR1 but not through TNFR2, due to species specificity of mouse TNFR2 [49]. Absence of TNFR1 from astrocytes, not neurons, abolished protection (Figure 7B). Together the results suggest that soluble hTNF engages astrocyte TNFR1, inducing activation and production of tmTNF. solTNF has been previously shown to increase the expression of tmTNF in cell lines [50]. Astrocyte tmTNF-neuronal TNFR2 interactions then precondition neurons for protection against glutamate excitotoxicity (Diagram 1).

To confirm the neuroprotective role of neuronal TNFR2, we compared pretreatment of WT astrocyteneuron co-cultures at NA-DIV7 with a novel TNFR2 agonist (TNFR2ag $100 \mathrm{ng} / \mathrm{ml}$ ) [51], or with soluble hTNF (100 ng/ml), for $24 \mathrm{~h}$ prior to NMDA challenge. As previously described [40, 52], TNFR2ag pretreatment induced strong neuroprotection against NMDA death, equal to that induced by soluble hTNF (Figure 7C). Together these results suggest that astrocyte tmTNF-neuronal TNFR2 interactions dominantly mediate preconditioning protection of neurons against glutamate excitotoxicity in vitro, and likely in vivo. Neuronal TNFR1 has limited neuroprotective effect in models of glutamate excitotoxicity.

\section{Discussion}

The TNF cytokine system is complex and associated with multiple functions in the CNS. It mediates overall neurotoxic and neuroprotective effects during CNS pathology depending on the cellular and molecular specificities of ligand-receptor interaction, and the underlying pathological mechanisms 
involved [1, 2]. To investigate the specific contributions of neuronal TNFR1 and TNFR2 to inflammatory and excitotoxic neurodegeneration processes relevant for MS, and to place these in the context of previous knowledge concerning neuroprotective effects of solTNF (and therefore TNFR1) in neuronal cultures in vitro $[14,15]$, we used mice in which TNFR1, TNFR2 or IKK $\beta$ was selectively depleted from excitatory glutamatergic neurons for experimental models of CNS demyelination and excitotoxicity, and astrocyte-neuron co-culture systems with different combinations of wild type, TNFKO, tmTNFKI, TNFR1KO and TNFR2KO cells in a glutamate excitotoxicity model.

The TNF system has well-described inflammatory and neurotoxic effects in the CNS. In vivo studies using conventional TNF or TNFR knockout mice, or TNF inhibitors, showed neurotoxic roles for solTNF and TNFR1 in a wide range of experimental models including ischemia-reperfusion retinal damage [17], spinal cord injury [53], Parkinson's disease [54], Alzheimer's disease [55], and MS [18, 19]. However, when TNFR1 is absent or solTNF is blocked in the entire organism, it is not possible to interpret the cellular mechanisms involved in neurotoxicity. A study of bone marrow chimeric mice generated using wild type and TNFR1KO bone marrow cells showed the essential TNFR1-responsive cells during EAE induction are CNS-resident cells [56]. Potent inflammatory effects of TNF, directly by activation of microglia, astrocytes, and endothelial cells, and indirectly through the induction of cell necroptosis and further neuroinflammation [57], are likely to dominate the neurotoxic effects of TNF in vivo. This is supported by the finding that NF-KB activation in nestin-expressing cells (astrocytes and neurons), and not OLG, determines neuroinflammation and myelin damage in the CPZ model [58].

Here we show that both neuronal TNFR1 and IKK $\beta$ accelerate microglia and astrocyte responses, demyelination and the onset of axon damage in the demyelination models, indicating common signaling pathways. Neuronal TNFR1 also induced the induction of autophagy in glial cells in the demyelinating lesions in the CPZ model, possibly in myelin-phagocytosing microglia, as recently described [46], and reflecting the increased demyelination and demand for clearance of myelin debris in control mice. Axonal damage and OLG loss were dependent upon the presence of neuronal TNFR1, not neuronal IKK $\beta$ during cuprizone demyelination, suggesting that other TNFR1 pathways signal axon and OLG pathology in this model. Several known TNF effects on neurons might be involved. First, by regulation of neuronal glutamate receptors. solTNF via TNFR1 rapidly $(15 \mathrm{~min})$ increases cell surface levels of $\mathrm{Ca}^{++}$-permeable GluR1-containing AMPAR in mouse hippocampal neurons, thereby enhancing synaptic strength at excitatory synapses in vitro [59], and potentiating excitotoxicity in vivo [60], [61, 62]. Similar effects are seen with NMDAR [63] [64]. Glutamate receptors and transporters are dysregulated during experimental demyelination in vivo [65], and might be sufficient to activate microglia in vivo in the context of disease and induce neuroinflammation. Second, by the induction of oxidative stress in neurons. Exposure of neurons to pathophysiological concentrations of solTNF results in rapid and profound decrease in mitochondrial function and reduced viability, an effect reduced by pretreatment of neurons with an antiTNFR1 antibody [66]. In the CPZ toxicity model, the function of many enzymes and mitochondria are already compromised by copper chelation, resulting in metabolic stress and the selective loss of OLG and demyelination [43]. Like OLG, neurons are also highly sensitive to oxidative stress. Damage of neuronal 
axons is a pathological feature of acute CPZ toxicity in young B6 mice and observed here in TNFR1ff control mice by increased numbers of APP-immunoreactive spheroids at the peak of demyelination. The observation that APP spheroids do not accumulate in nTNFR1KO mice indicates that the combination of neuronal TNFR1 and CPZ causes direct damage in neurons and their axons.

The TNF system also has prominent neuroprotective roles in the CNS. One of the first reported effects of solTNF (and therefore TNFR1) in vitro was neuroprotection. Pretreatment of primary neuron cultures with solTNF protected them against a variety of metabolic-excitotoxic-oxidative insults and promoted maintenance of calcium homeostasis $[14,15]$. Neuroprotective effects of TNFR1 and TNFR2 were also observed in specific in vivo experimental paradigms including cerebral stroke [16] and glutamate excitotoxicity [40,52], but the cellular mechanisms of these effects is poorly understood. Recently, the availability of mouse lines carrying conditional TNFR1 [27] or TNFR2 [28] alleles made investigation of cell-specific roles of the TNFR in CNS possible. An elegant study using mice with myeloid cell-specific ablation of TNFR2, shows that microglial TNFR2 protects mice against the initiation of EAE [20]. Another study using mice with OLG lineage cell-specific ablation of TNFR2 shows that OLG TNFR2 ameliorates clinical symptoms particularly in the chronic phase of EAE, reduces myelin and axonal pathology, drives the differentiation of OLG precursor cells into mature OLG, and improves remyelination [21]. The effects of OLG-specific TNFR2 depletion in EAE largely recapitulate the effects of total TNFR2 KO in CPZ demyelination [12] suggesting that protective effects of TNFR2 in models of inflammatory demyelination are mediated predominantly by microglia and OLG. The finding here that neuronal TNFR2 did not alter the development of EAE or CPZ demyelination further supports this conclusion. Neuronal TNFR2 did however play a role in the inducton of preconditioning protection against seizures in a KA excitotoxicity model. Also, neuronal TNFR2 protected hippocampal neurons against excitotoxic death in control mice.

Together, our results show that neuronal TNFR1 and IKK $\beta$ promote neuroinflammation and the onset of demyelination in mouse models of MS, and that neuronal TNFR1 is additionally required for axon damage, OLG loss and induction of glial cell autophagy in white matter lesions during CPZ demyelination, possibly by increasing neuronal oxidative stress. On the contrary, neuronal TNFR2 had no detectable effects on the clinical or histopathological features of the MS models, but our results provide the first evidence that TNFR2 directly protects neurons in vivo under conditions of glutamate excitotoxicity that are relevant for a wide range of CNS diseases. Also, the previously reported neuroprotective effects of solTNF (and therefore TNFR1) in vitro were reconciled here with in vivo findings, with evidence from astrocyte-neuron co-cultures that astrocyte TNFR1 and tmTNF, and neuronal TNFR2 are necessary, and that a TNFR2 agonist is sufficient, to mediate TNF preconditioning neuroprotection against glutamate excitotoxicity in vitro. This is consistent with previous findings with TNFR2 agonists, which protected human dopaminergic neuronal cells against oxidative stress via activation of the PI3K-PKB/Akt pathway, or challenge with 6-OHDA in vitro [67], and enriched mouse cortical neurons against glutamate excitotoxicity [52].

\section{Conclusions}


The results of this study show that the neuronal TNFR have fundamentally different roles in CNS pathology in vivo, with signaling through neuronal TNFR1 and IKK $\beta$ promoting microglia responses and neurotoxicity in mouse demyelination models, and neuronal TNFR2 being involved in preconditioning protection in excitotoxicity, but not in inflammatory demyelination. Details of the molecular and cellular mechanisms underlying previously described neuroprotective properties of solTNF in vitro were obtained using astrocyte-neuron co-cultures with different combinations of wildtype and knockout cells. Results further supported the in vivo findings, with solTNF (and therefore TNFR1) indirectly protecting neurons against NMDA excitotoxicity via astrocyte tmTNF- neuronal TNFR2 interactions, and being fully reproduced by a TNFR2 agonist. Together the results consolidate the concept that selective inhibition of solTNF/TNFR1 with maintenance or stimulation of beneficial TNFR2 function represents a promising strategy for suppressing inflammation and maintaining neuroprotection for the treatment of a wide range of CNS diseases.

\section{Abbreviations}

AIF, apoptosis inducing factor

APP, amyloid precursor protein

B6, C57BL/6 mice

$\mathrm{C} / 2$, mouse $\mathrm{C}-\mathrm{C}$ motif chemokine ligand 2 gene

CFA, complete Freund's adjuvant

CNPase, 2', 3'-cyclic nucleotide 3'-phosphodiesterase

CPZ, cuprizone

CPZn, weeks of cuprizone feeding

CNS, central nervous system

Cre, Cre recombinase from bacteriophage P1

Cxcl16, mouse $\mathrm{C}-\mathrm{X}-\mathrm{C}$ motif chemokine ligand 16 gene

DAB, 3'3'-diaminobenzidine

DIVn, number of days in vitro

En, embryonic day

EAE, experimental autoimmune encephalomyelitis 
ff, LoxP-flanked DNA sequence

GFAP, glial fibrillary acidic protein

Gusb, mouse glucuronidase beta gene

$H 2-A b 1$, mouse $\mathrm{H}-2$ class II histocompatibility antigen, $\mathrm{A}$ beta chain gene

hTNF, human tumor necrosis factor

Iba1, ionized calcium-binding adaptor molecule 1

IHC, immunohistochemistry

IKK $\beta$, inhibitor of nuclear factor kappa-B kinase subunit beta

IKKßff, littermate control mice with non-deleted LoxP-flanked IKKß sequence

KA, kainic acid

$\mathrm{KI}$, knockin

KO, knockout

LC3, MAP1-LC3 structural protein of autophagosomal membrane

LFB, Klüver-Barrera Luxol fast blue

LoxP, recognition sequence for Cre from bacteriophage P1

$M b p$, mouse myelin basic protein gene

MK801, NMDA receptor antagonist

MOG, murine myelin oligodendrocyte glycoprotein

MS, multiple sclerosis

NeuN, neuronal nuclear antigen

NF-KB, Nuclear Factor kappa-light-chain-enhancer of activated B cells

NA-DIVn, neuron-astrocyte co-cultures number of days in vitro

NMDA, N-methyl-D-aspartate

nTNFR1/2KO, neuron-specific TNFR1/2 knockout mice 
Olig2, mouse oligodendrocyte transcription factor 2 gene

OPC, oligodendrocyte precursor cells

PCR, polymerase chain reaction

PI3K-PKB/Akt, phosphatidylinositol 3-kinase and Akt/protein kinase B signalling pathway

PTx, Bordetella pertussis toxin

S.c., subcutaneous

SMI 31, neurofilament H phosphorylated

Snap25, mouse synaptosomal-associated protein 25kDa gene

solTNF, soluble TNF

tmTNF, transmembrane TNF

TNF, tumor necrosis factor

TNFR1/2ff, littermate control mice with non-deleted LoxP-flanked TNFR1/2 sequences

TNFR1, TNF receptor 1

TNFR2, TNF receptor 2

WT, wildtype mice.

\section{Declarations}

\section{Ethical Approval}

The animal study was reviewed and approved by the Committee for Evaluation of Experimental Procedures, Department of Experimental Animal Models, Hellenic Pasteur Institute (Presided by Dr P Andriopoulos, pandriopoulos@patt.gov.gr, for the Hellenic Republic, General Secretariat for Agricultural Economy, Veterinary and Licenses). License numbers 4457/10-07-2014 and 2579/31-05-2018

\section{Consent for publication}

Not applicable.

\section{Availability of supporting data}

The datasets used and/or analysed during the current study are available from the corresponding author on reasonable request. 


\section{Competing interests}

The authors declare that they have no competing interests.

\section{Funding}

This research was co-financed by European Union and Greek national funds by The Management and Implementation Authority for Research, Technological Development and Innovation Actions (MIA-RTDI/ EYAE-ETAK) of the Hellenic Ministry of Development and Investments, through the Operational Program Competitiveness, Entrepreneurship and Innovation, under the call RESEARCH - CREATE - INNOVATE (project code T1EDK-01859, acronym AKESO).

\section{Authors contributions}

IP designed and performed research, analyzed data and contributed to writing the paper; $E T, M K, A B$ and $\mathrm{LI}$ performed research and analyzed data; KK analyzed and interpreted data; RF and REK contributed unpublished reagents; MD and GK contributed unpublished analytical tools; HL performed research, analyzed and interpreted data and contributed to writing the paper; LP designed research, analyzed and interpreted data, and wrote the paper. All authors edited the final manuscript.

\section{Acknowledgements}

We wish to thank Ulrich Eisel for advice in setting up the cortical neuronal cultures, Eirini Fragkiadaki responsible DVM (MSc, PhD -FELASA accredited B, C, D), and the personnel of the Department of Animal Models for Biomedical Research of Hellenic Pasteur Institute, Athens, Greece for assisting during mice handling and maintenance. We also thank Bernhard Ryffel, CNRS, laboratory of Molecular Immunology and Embryology, Orleans, France for providing tmTnf $\Delta / \Delta$ (tmTNFKI) mice, Horst Bluethmann, F. Hoffmann-La Roche Ltd, Basel, Switzerland for Tnfrsf1a-/-(TNFR1KO) mice and Michael Karin, Department of Pharmacology, University of California at San Diego, California, USA for $I k k \beta^{F / F}$ (IKK $\left.\beta f f\right)$ mice.

\section{Authors information}

LI current address is: Institute of Immunology, Biomedical Sciences Research Centre (BSRC) "Alexander Fleming," Vari 16672, Athens, Greece.

\section{References}

1. Olmos, G. and J. Llado, Tumor necrosis factor alpha: a link between neuroinflammation and excitotoxicity. Mediators Inflamm, 2014. 2014: p. 861231.

2. Probert, L., TNF and its receptors in the CNS: The essential, the desirable and the deleterious effects. Neuroscience, 2015. 302: p. 2-22. 
3. van Oosten, B.W., et al., Increased MRI activity and immune activation in two multiple sclerosis patients treated with the monoclonal anti-tumor necrosis factor antibody cA2. Neurology, 1996. 47(6): p. 1531-4.

4. Mohan, N., et al., Demyelination occurring during anti-tumor necrosis factor a therapy for inflammatory arthritides. Arthritis \& Rheumatism, 2001. 44(12): p. 2862-2869.

5. Antonazzo, I.C., et al., Multiple sclerosis as an adverse drug reaction: clues from the FDA Adverse Event Reporting System. Expert Opin Drug Saf, 2018. 17(9): p. 869-874.

6. De Jager, P.L., et al., Meta-analysis of genome scans and replication identify CD6, IRF8 and TNFRSF1A as new multiple sclerosis susceptibility loci. Nat Genet, 2009. 41(7): p. 776-82.

7. Gregory, A.P., et al., TNF receptor 1 genetic risk mirrors outcome of anti-TNF therapy in multiple sclerosis. Nature, 2012. 488(7412): p. 508-511.

8. Medler, J. and H. Wajant, Tumor necrosis factor receptor-2 (TNFR2): an overview of an emerging drug target. Expert Opin Ther Targets, 2019. 23(4): p. 295-307.

9. Brambilla, R., et al., Inhibition of soluble tumour necrosis factor is therapeutic in experimental autoimmune encephalomyelitis and promotes axon preservation and remyelination. Brain, 2011. 134(Pt 9): p. 2736-54.

10. Taoufik, E., et al., Transmembrane tumour necrosis factor is neuroprotective and regulates experimental autoimmune encephalomyelitis via neuronal nuclear factor-kappaB. Brain, 2011. 134(Pt 9): p. 2722-35.

11. Karamita, M., et al., Therapeutic inhibition of soluble brain TNF promotes remyelination by increasing myelin phagocytosis by microglia. JCI Insight, 2017. 2(8).

12. Arnett, H.A., et al., TNF alpha promotes proliferation of oligodendrocyte progenitors and remyelination. Nat Neurosci, 2001. 4(11): p. 1116-22.

13. Pegoretti, V., et al., Selective Modulation of TNF-TNFRs Signaling: Insights for Multiple Sclerosis Treatment. Front Immunol, 2018. 9: p. 925.

14. Cheng, B., S. Christakos, and M.P. Mattson, Tumor necrosis factors protect neurons against metabolic-excitotoxic insults and promote maintenance of calcium homeostasis. Neuron, 1994. 12(1): p. 139-53.

15. Barger, S.W., et al., Tumor necrosis factors alpha and beta protect neurons against amyloid betapeptide toxicity: evidence for involvement of a kappa B-binding factor and attenuation of peroxide and Ca2+ accumulation. Proc Natl Acad Sci U S A, 1995. 92(20): p. 9328-32.

16. Gary, D.S., et al., Ischemic and excitotoxic brain injury is enhanced in mice lacking the $p 55$ tumor necrosis factor receptor. J Cereb Blood Flow Metab, 1998. 18(12): p. 1283-7.

17. Fontaine, V., et al., Neurodegenerative and neuroprotective effects of tumor Necrosis factor (TNF) in retinal ischemia: opposite roles of TNF receptor 1 and TNF receptor 2. J Neurosci, 2002. 22(7): p. Rc216. 
18. Eugster, H.P., et al., Severity of symptoms and demyelination in MOG-induced EAE depends on TNFR1. Eur J Immunol, 1999. 29(2): p. 626-32.

19. Suvannavejh, G.C., et al., Divergent roles for $p 55$ and $p 75$ tumor necrosis factor receptors in the pathogenesis of MOG(35-55)-induced experimental autoimmune encephalomyelitis. Cell Immunol, 2000. 205(1): p. 24-33.

20. Gao, H., et al., Opposing Functions of Microglial and Macrophagic TNFR2 in the Pathogenesis of Experimental Autoimmune Encephalomyelitis. Cell reports, 2017. 18(1): p. 198-212.

21. Madsen, P.M., et al., Oligodendroglial TNFR2 Mediates Membrane TNF-Dependent Repair in Experimental Autoimmune Encephalomyelitis by Promoting Oligodendrocyte Differentiation and Remyelination. J Neurosci, 2016. 36(18): p. 5128-43.

22. Taniguchi, K. and M. Karin, NF-kappaB, inflammation, immunity and cancer: coming of age. Nat Rev Immunol, 2018. 18(5): p. 309-324.

23. Pasparakis, M., et al., Immune and inflammatory responses in TNF alpha-deficient mice: a critical requirement for TNF alpha in the formation of primary $B$ cell follicles, follicular dendritic cell networks and germinal centers, and in the maturation of the humoral immune response. J Exp Med, 1996. 184(4): p. 1397-411.

24. Ruuls, S.R., et al., Membrane-bound TNF supports secondary lymphoid organ structure but is subservient to secreted TNF in driving autoimmune inflammation. Immunity, 2001. 15(4): p. 533-43.

25. Rothe, J., et al., Mice lacking the tumour necrosis factor receptor 1 are resistant to TNF-mediated toxicity but highly susceptible to infection by Listeria monocytogenes. Nature, 1993. 364(6440): p. 798-802.

26. Erickson, S.L., et al., Decreased sensitivity to tumour-necrosis factor but normal T-cell development in TNF receptor-2-deficient mice. Nature, 1994. 372(6506): p. 560-3.

27. Van Hauwermeiren, F., et al., Safe TNF-based antitumor therapy following p55TNFR reduction in intestinal epithelium. J Clin Invest, 2013. 123(6): p. 2590-603.

28. Sakkou, M., et al., Mesenchymal TNFR2 promotes the development of polyarthritis and comorbid heart valve stenosis. JCI Insight, 2018. 3(7).

29. Park, J.M., et al., Macrophage apoptosis by anthrax lethal factor through p38 MAP kinase inhibition. Science, 2002. 297(5589): p. 2048-51.

30. Emmanouil, M., et al., Neuronal I kappa B kinase beta protects mice from autoimmune encephalomyelitis by mediating neuroprotective and immunosuppressive effects in the central nervous system. J Immunol, 2009. 183(12): p. 7877-89.

31. Evangelidou, M., et al., Altered expression of oligodendrocyte and neuronal marker genes predicts the clinical onset of autoimmune encephalomyelitis and indicates the effectiveness of multiple sclerosis-directed therapeutics. J Immunol, 2014. 192(9): p. 4122-33.

32. Hiremath, M.M., et al., Microglial/macrophage accumulation during cuprizone-induced demyelination in C57BL/6 mice. J Neuroimmunol, 1998. 92(1-2): p. 38-49. 
33. Hatazaki, S., et al., Microarray profile of seizure damage-refractory hippocampal CA3 in a mouse model of epileptic preconditioning. Neuroscience, 2007. 150(2): p. 467-77.

34. Schauwecker, P.E. and O. Steward, Genetic determinants of susceptibility to excitotoxic cell death: implications for gene targeting approaches. Proc Natl Acad Sci U S A, 1997. 94(8): p. 4103-8.

35. Racine, R.J., Modification of seizure activity by electrical stimulation. II. Motor seizure. Electroencephalogr Clin Neurophysiol, 1972. 32(3): p. 281-94.

36. Evangelidou, M., et al., Altered Expression of Oligodendrocyte and Neuronal Marker Genes Predicts the Clinical Onset of Autoimmune Encephalomyelitis and Indicates the Effectiveness of Multiple Sclerosis-Directed Therapeutics. The Journal of Immunology, 2014: p. 1300633.

37. Daskalakis, K., et al., Increased autophagy/mitophagy levels in primary tumours of patients with pancreatic neuroendocrine neoplasms. Endocrine, 2020. 68(2): p. 438-447.

38. Alissafi, T., et al., Tregs restrain dendritic cell autophagy to ameliorate autoimmunity. J Clin Invest, 2017. 127(7): p. 2789-2804.

39. Taoufik, E., et al., $F L I P(L)$ protects neurons against in vivo ischemia and in vitro glucose deprivationinduced cell death. J Neurosci, 2007. 27(25): p. 6633-46.

40. Marchetti, L., et al., Tumor necrosis factor (TNF)-mediated neuroprotection against glutamateinduced excitotoxicity is enhanced by $N$-methyl-D-aspartate receptor activation. Essential role of a TNF receptor 2-mediated phosphatidylinositol 3-kinase-dependent NF-kappa B pathway. J Biol Chem, 2004. 279(31): p. 32869-81.

41. Fujikawa, D.G., The role of excitotoxic programmed necrosis in acute brain injury. Computational and structural biotechnology journal, 2015. 13: p. 212-221.

42. Mahad, D.H., B.D. Trapp, and H. Lassmann, Pathological mechanisms in progressive multiple sclerosis. Lancet Neurol, 2015. 14(2): p. 183-93.

43. Praet, J., et al., Cellular and molecular neuropathology of the cuprizone mouse model: clinical relevance for multiple sclerosis. Neurosci Biobehav Rev, 2014. 47: p. 485-505.

44. Yuan, Y., et al., TNF-a induces autophagy through ERK1/2 pathway to regulate apoptosis in neonatal necrotizing enterocolitis model cells IEC-6. Cell Cycle, 2018. 17(11): p. 1390-1402.

45. Azad, M.B., Y. Chen, and S.B. Gibson, Regulation of autophagy by reactive oxygen species (ROS): implications for cancer progression and treatment. Antioxid Redox Signal, 2009. 11(4): p. 777-90.

46. Berglund, R., et al., Microglial autophagy-associated phagocytosis is essential for recovery from neuroinflammation. Sci Immunol, 2020. 5(52).

47. Kang, Z., et al., IL-17-induced Act1-mediated signaling is critical for cuprizone-induced demyelination. J Neurosci, 2012. 32(24): p. 8284-92.

48. Cheng, B. and M.P. Mattson, NT-3 and BDNF protect CNS neurons against metabolic/excitotoxic insults. Brain Res, 1994. 640(1-2): p. 56-67.

49. Lewis, M., et al., Cloning and expression of cDNAs for two distinct murine tumor necrosis factor receptors demonstrate one receptor is species specific. Proceedings of the National Academy of 
Sciences, 1991. 88(7): p. 2830-2834.

50. Grell, M., et al., Induction of cell death by tumour necrosis factor (TNF) receptor 2, CD40 and CD30: a role for TNF-R1 activation by endogenous membrane-anchored TNF. Embo j, 1999. 18(11): p. 303443.

51. Fischer, R., et al., Novel strategies to mimic transmembrane tumor necrosis factor-dependent activation of tumor necrosis factor receptor 2. Scientific Reports, 2017. 7(1): p. 6607.

52. Dong, Y., et al., Essential protective role of tumor necrosis factor receptor 2 in neurodegeneration. Proc Natl Acad Sci U S A, 2016. 113(43): p. 12304-12309.

53. Novrup, H.G., et al., Central but not systemic administration of XPro1595 is therapeutic following moderate spinal cord injury in mice. Journal of Neuroinflammation, 2014. 11(1): p. 159.

54. McCoy, M.K., et al., Blocking soluble tumor necrosis factor signaling with dominant-negative tumor necrosis factor inhibitor attenuates loss of dopaminergic neurons in models of Parkinson's disease. J Neurosci, 2006. 26(37): p. 9365-75.

55. McAlpine, F.E., et al., Inhibition of soluble TNF signaling in a mouse model of Alzheimer's disease prevents pre-plaque amyloid-associated neuropathology. Neurobiol Dis, 2009. 34(1): p. 163-77.

56. Gimenez, M.A., et al., A Tumor Necrosis Factor Receptor 1-Dependent Conversation between Central Nervous System-Specific T Cells and the Central Nervous System Is Required for Inflammatory Infiltration of the Spinal Cord. The American Journal of Pathology, 2006. 168(4): p. 1200-1209.

57. Yuan, J., P. Amin, and D. Ofengeim, Necroptosis and RIPK1-mediated neuroinflammation in CNS diseases. Nat Rev Neurosci, 2019. 20(1): p. 19-33.

58. Raasch, J., et al., IkappaB kinase 2 determines oligodendrocyte loss by non-cell-autonomous activation of NF-kappaB in the central nervous system. Brain, 2011. 134(Pt 4): p. 1184-98.

59. Beattie, E.C., et al., Control of synaptic strength by glial TNFalpha. Science, 2002. 295(5563): p. 22825.

60. Leonoudakis, D., P. Zhao, and E.C. Beattie, Rapid tumor necrosis factor alpha-induced exocytosis of glutamate receptor 2-lacking AMPA receptors to extrasynaptic plasma membrane potentiates excitotoxicity. J Neurosci, 2008. 28(9): p. 2119-30.

61. Bernardino, L., et al., Modulator effects of interleukin-1beta and tumor necrosis factor-alpha on AMPA-induced excitotoxicity in mouse organotypic hippocampal slice cultures. J Neurosci, 2005. 25(29): p. 6734-44.

62. Stellwagen, D., et al., Differential regulation of AMPA receptor and GABA receptor trafficking by tumor necrosis factor-alpha. J Neurosci, 2005. 25(12): p. 3219-28.

63. Wheeler, D., et al., Tumor necrosis factor-alpha-induced neutral sphingomyelinase-2 modulates synaptic plasticity by controlling the membrane insertion of NMDA receptors. J Neurochem, 2009. 109(5): p. 1237-49.

64. Yin, X., et al., Regulation of NMDA receptor transport: a KIF17-cargo binding/releasing underlies synaptic plasticity and memory in vivo. J Neurosci, 2012. 32(16): p. 5486-99. 
65. Azami Tameh, A., et al., Regional regulation of glutamate signaling during cuprizone-induced demyelination in the brain. Ann Anat, 2013. 195(5): p. 415-23.

66. Doll, D.N., et al., Rapid mitochondrial dysfunction mediates TNF-alpha-induced neurotoxicity. J Neurochem, 2015. 132(4): p. 443-51.

67. Fischer, R., et al., A TNF receptor 2 selective agonist rescues human neurons from oxidative stressinduced cell death. PLoS One, 2011. 6(11): p. e27621.

\section{Figures}


A.

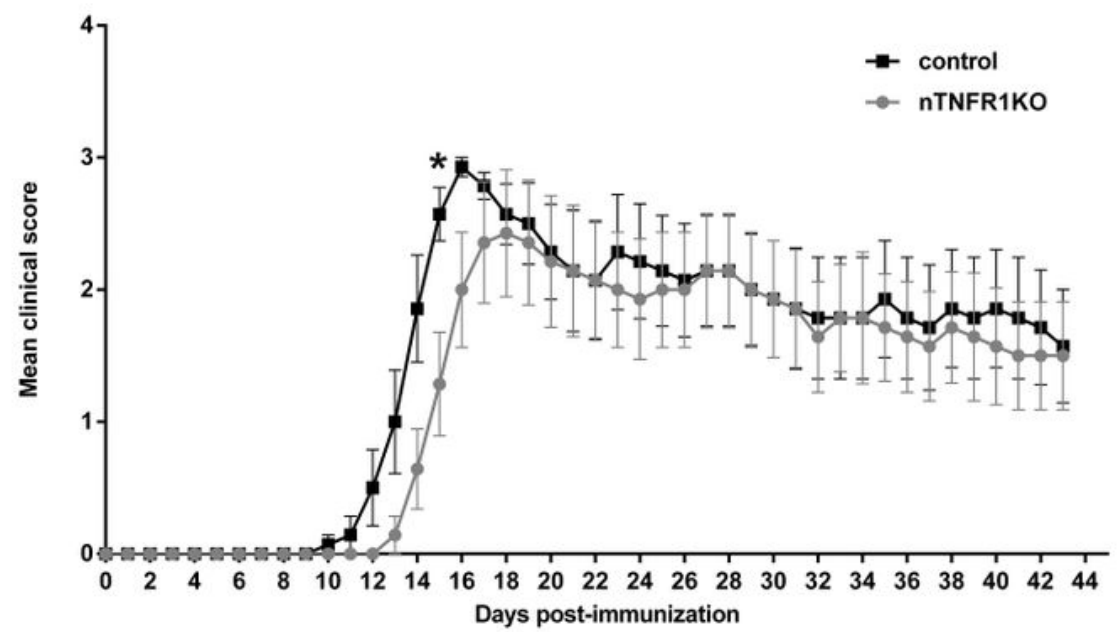

B.
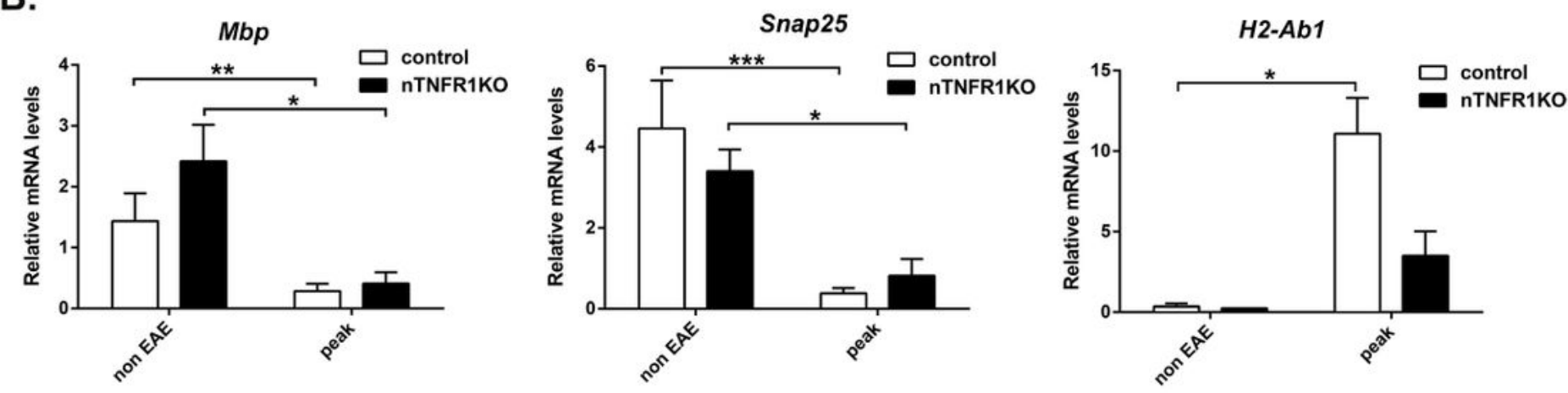

C.

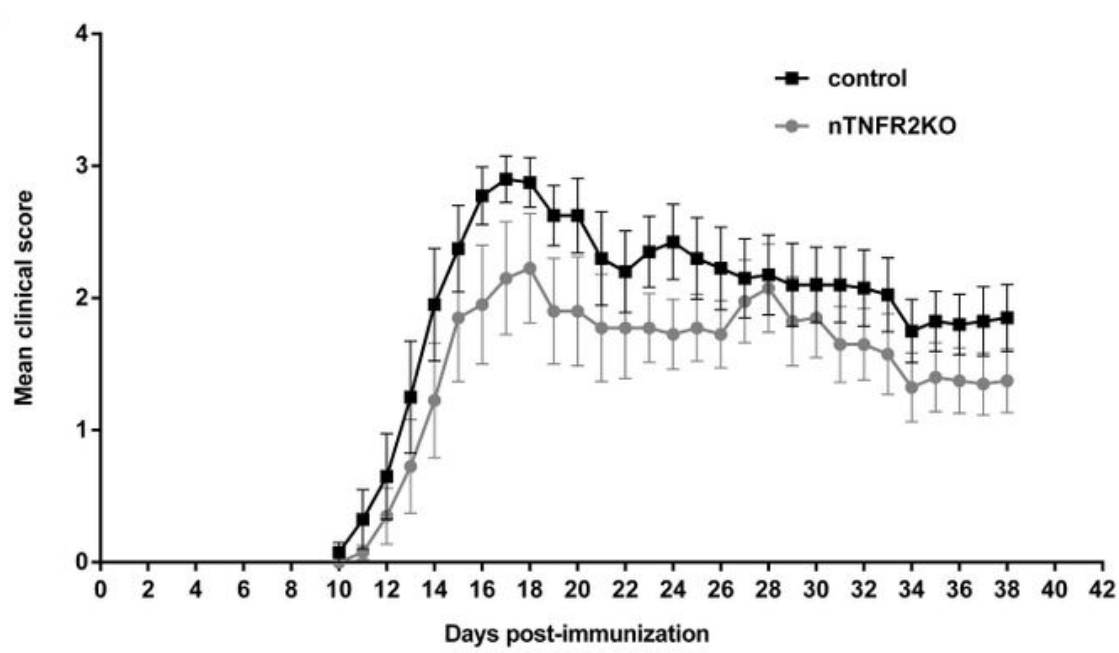

\section{Figure 1}

Neuronal TNFR1 advances the onset of clinical symptoms and spinal cord inflammation in EAE. (A) Mean clinical scores for $n$ TNFR1KO $(n=7)$ and control (TNFR1ff, $n=7)$ mice after immunization with MOG/CFA/PTx. (B) Differential expression of myelin, neuronal and immune genes relative to GusB in total mRNA isolates taken from nTNFR1KO and control spinal cords of non-immunized (non-EAE) and immunized mice at peak of EAE in the control group (peak) (non-EAE: control, $n=3$ and nTNFR1KO, n=4; 
peak EAE: control, $n=10$ and nTNFR1KO, $n=3)$. (C) Mean clinical scores for nTNFR2KO $(n=10)$ and control (TNFR2ff, $n=10$ ) mice after immunization with MOG/CFA/PTx. Results shown are representative of two $(A)$ or three (C) independent experiments and are presented as mean values \pm SEM. Statistical significance after pairwise comparisons between measurements of EAE clinical scores between the KO and control mouse strains $(A, C)$ and mRNA levels $(B)$ are shown by Student's t-test. * $p \leq 0.05,{ }^{* *} p \leq$ $0.005, * * * \mathrm{p} \leq 0.001$.
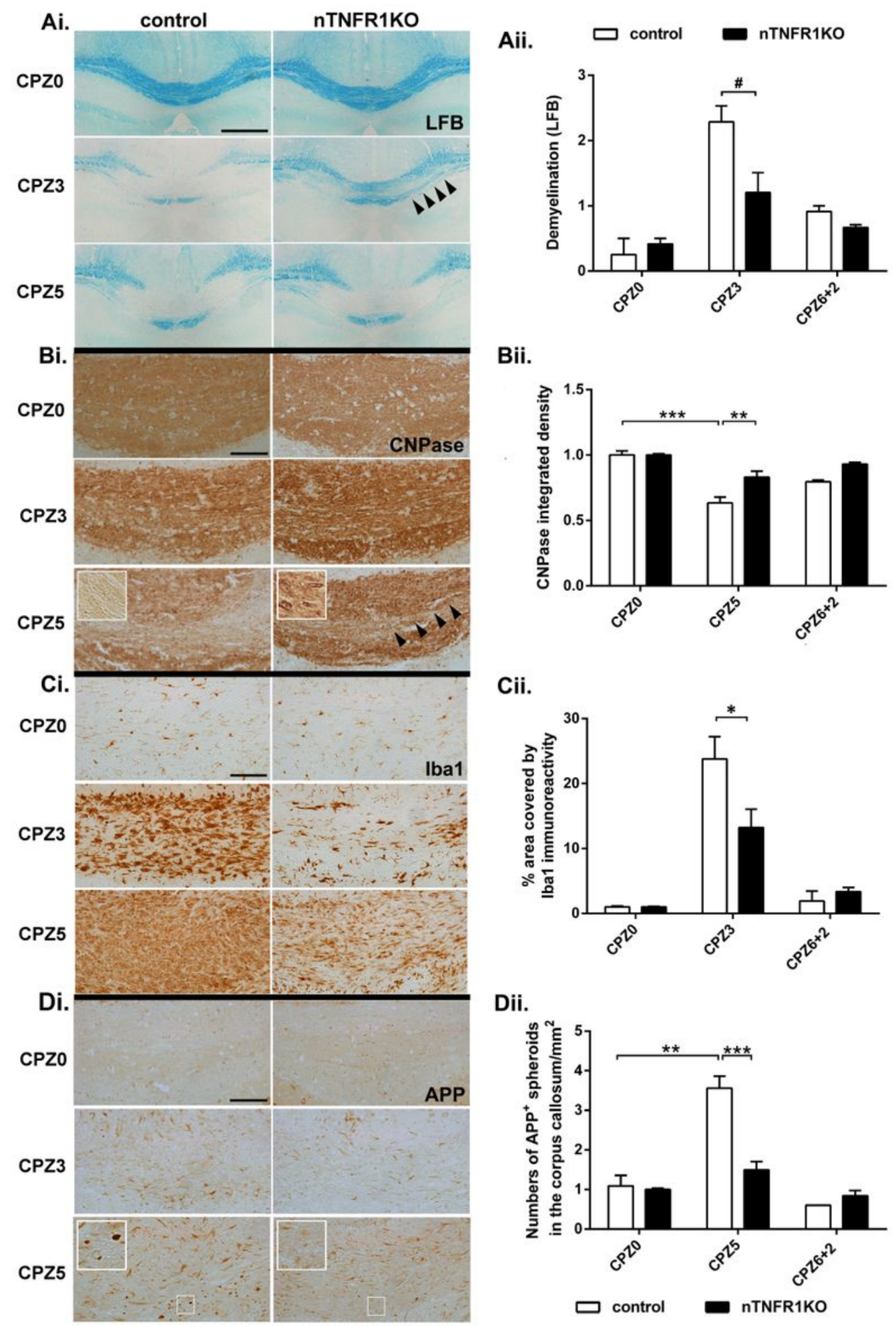

Bii.

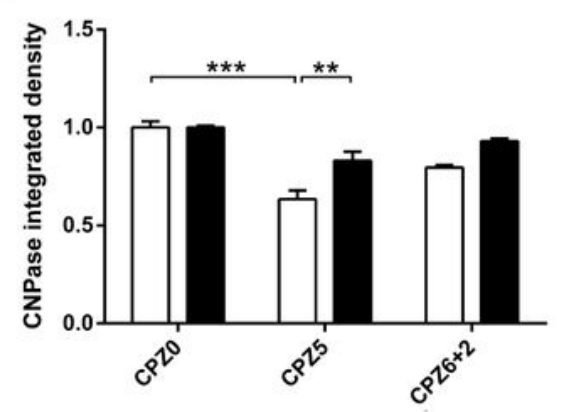

Cii.

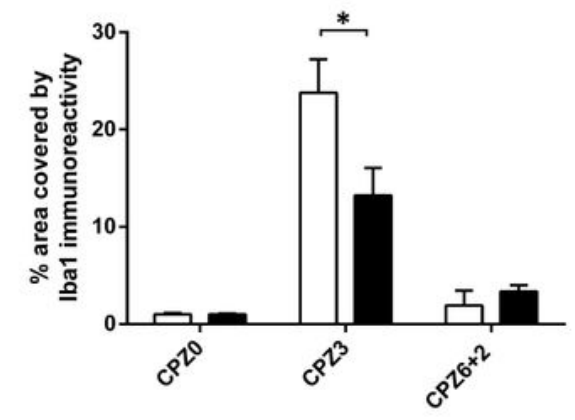

Dii.

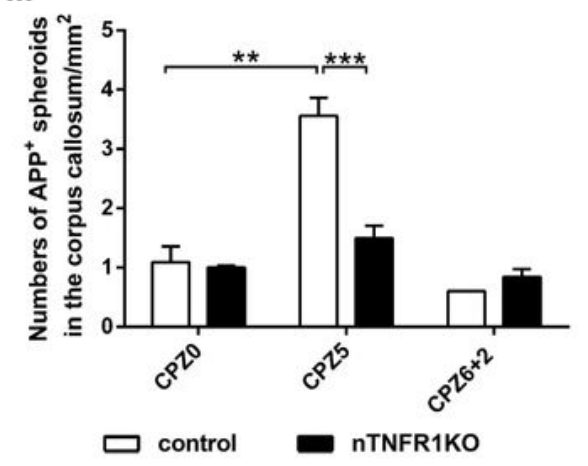

Figure 2 
Neuronal TNFR1 promotes microglial responses, oligodendrocyte loss and axon damage in cuprizone demyelination. (Ai) LFB staining of myelin (arrowheads showing maintenance of myelin at CPZ3 in nTNFR1KO mice), (Bi) CNPase immunostaining of myelin and OLG (arrowheads and inset showing maintenance of myelin and OLG at CPZ5 in nTNFR1KO mice), (Ci) Iba1 immunostaining of microglia, and (Di) APP immunostaining of axonal spheroids (inset showing higher magnification) in serial brain coronal paraffin sections through the medial corpus callosum from representative nTNFR1KO and TNFR1ff control naïve (CPZ0) or CPZ-fed CPZ3, CPZ5 and CPZ6+2 mice. Scale bars: $500 \mu \mathrm{M}(\mathrm{Ai})$, and $100 \mu \mathrm{M}(\mathrm{Bi}, \mathrm{Ci}, \mathrm{Di})$. (Aii) Semiquantitative scoring of demyelination (loss of LFB staining) in the corpus callosum of the same groups of mice represented in Ai. Quantitative representation of: (Bii) CNPase immunoreactivity by densitometry, (Cii) Iba1 immunoreactivity by \% area covered, and (Dii) numbers of APP immunoreactive spheroids/mm2 tissue in the corpus callosum of groups of mice represented in $\mathrm{Bi}$, $\mathrm{Ci}$, and $\mathrm{Di}$, respectively. Results shown in Bii and Dii are normalized against values in CPZO mice of each genotype ( $n=2$ for all CPZO and control $C P Z 6+2$, and $n \geq 7$ mice for other time points). Results are from two independent experiments. The most relevant statistically significant differences after comparisons between groups are shown by two-way ANOVA with Bonferroni's test $\left({ }^{*}\right)$, and pairwise comparisons by Student's t-test (\#). *, \#p $\leq 0.05,{ }^{* *} p \leq 0.005,{ }^{* * *} p \leq 0.001$.
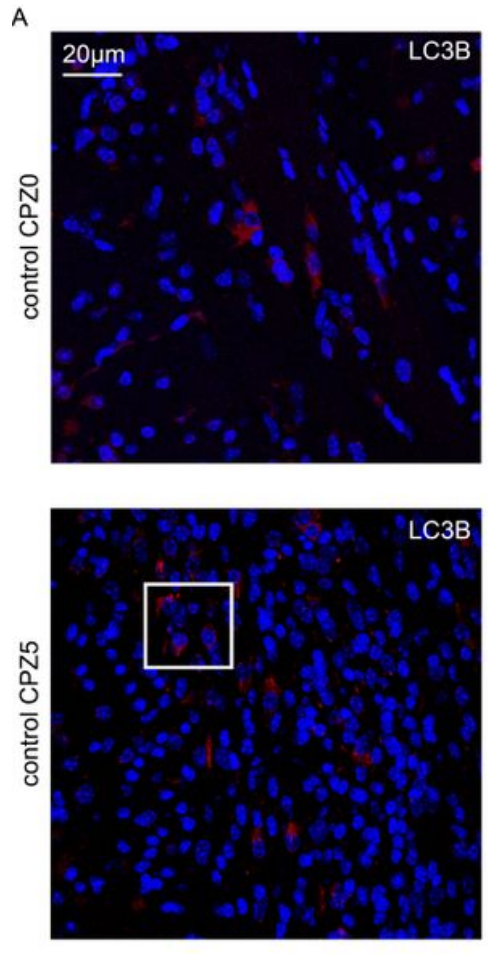
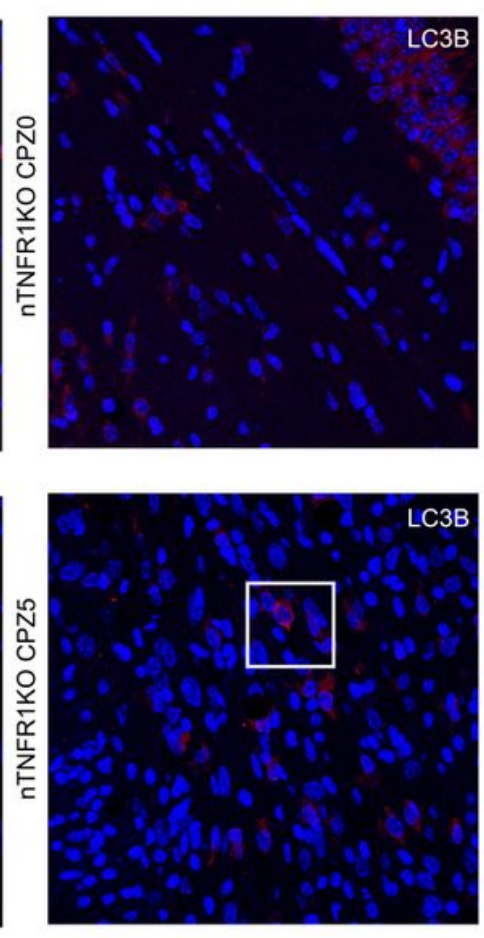
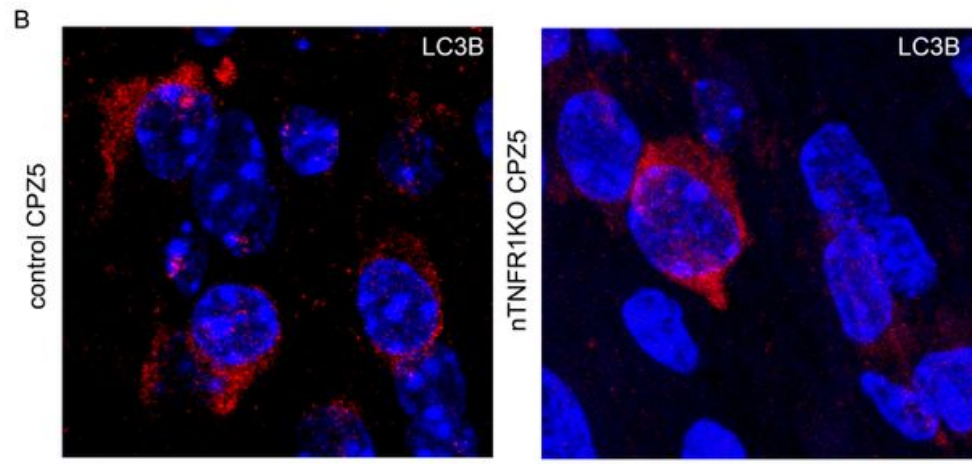

C

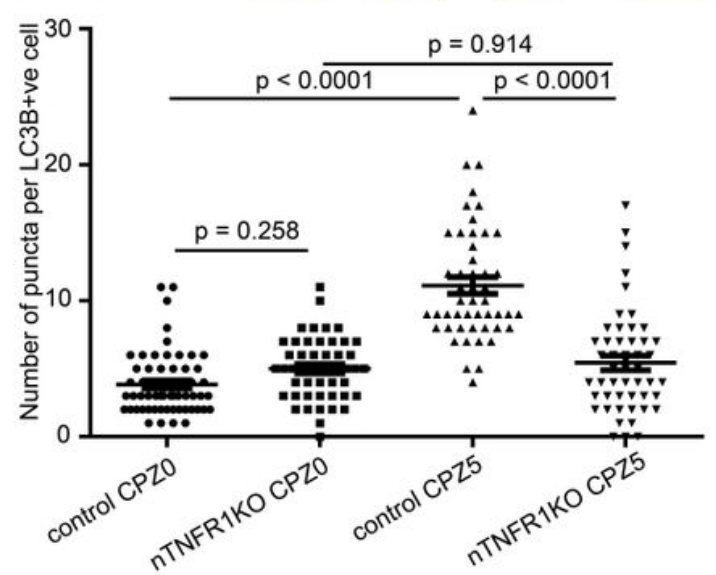

\section{Figure 3}

Neuronal TNFR1 increases the induction of autophagy in corpus callosum glial cells. (A) Immunofluorescence staining of LC3B (red), and DAPI (blue) in brain coronal paraffin sections through the medial corpus callosum from representative naïve (CPZ0) and CPZ-fed CPZ5 mice of each genotype ( $n=3$ for all CPZO and CPZ5 time points). Scale bars: $20 \mu \mathrm{m} ; 60$ x objective. (B) Quantitative analysis of 
LC3B-positive puncta in LC3-immunoreactive cells ( $n=50$ LC3B-immunoreactive cells were measured from each mouse strain at each time point). Means \pm SD LC3B puncta/cell are depicted. $p$-value has been calculated by one-way ANOVA

Ai.

CPZO

CPZ5

Ci.

control

nIKKßKO
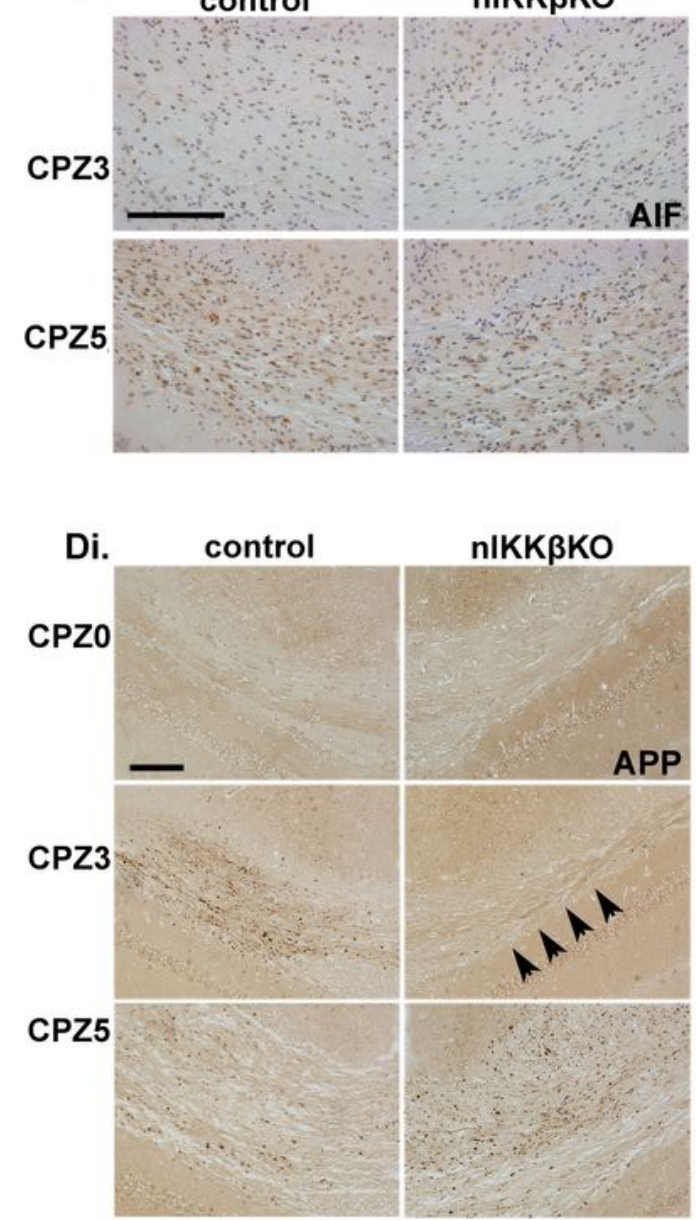

Aii.

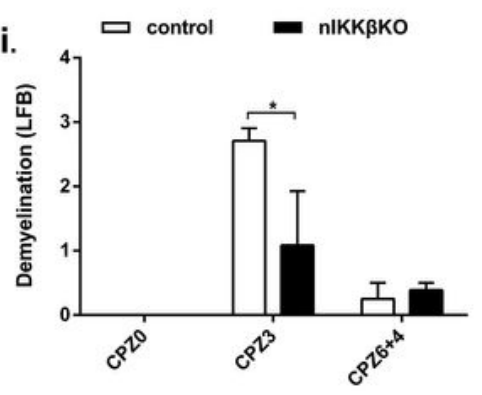

B.

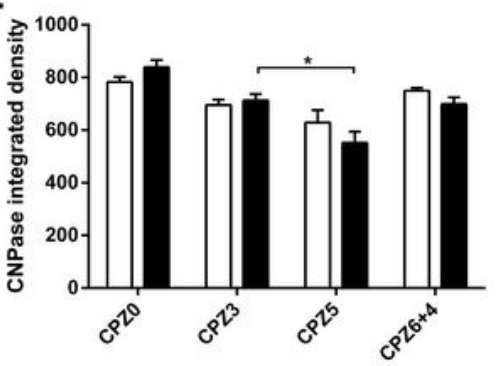

Cii.

D
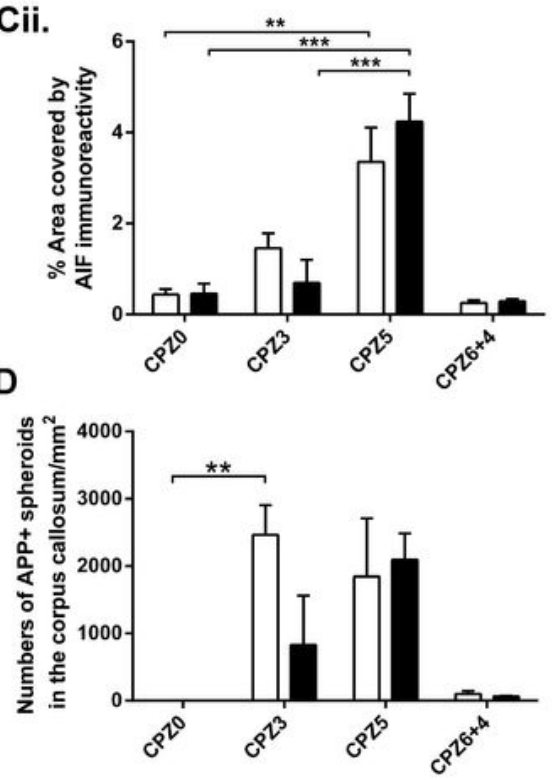

E.

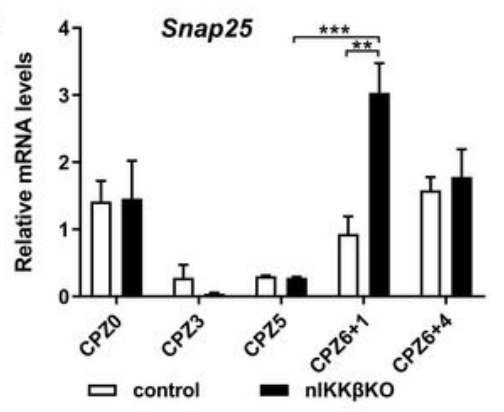

Figure 4

Neuronal IKK $\beta$ promotes the onset of cuprizone demyelination and axon damage. (Ai) LFB staining of myelin (arrowheads showing maintenance of myelin at CPZ3 in nIKKßKO mice), (Ci) apoptosis inducing 
factor (AIF) immunostaining of apoptotic cells, and (Di) APP immunostaining of axonal spheroids in serial brain coronal paraffin sections from nIKKKKO and IKKßff control naïve (CPZO) or CPZ-fed CPZ3, $\mathrm{CPZ} 5$ and $\mathrm{CPZ6}+4$ mice (arrows shows the preservation of myelin (Ai) and reduced APP plaques (Di) in nIKK KKO mice at CPZ3). Scale bars: $100 \mu \mathrm{M}$. (Aii) Semiquantitative scoring of demyelination (loss of LFB staining), and quantitative representation of (B) CNPase immunoreactivity of myelin and OLG by densitometry, (Cii) AIF immunoreactivity by \% area covered, and (Dii) numbers of APP spheroids/mm2 tissue in the corpus callosum in groups of nIKKKKO and control mice represented in the photographs. (E) Differential expression of neuronal Snap25 relative to GusB in total mRNA isolates taken from nIKK $\beta K O$ and IKK $3 f f$ control brains from CPZ0 or CPZ-fed CPZ3, CPZ5, CPZ6+1 and CPZ6+4 mice. Results are means of $2(\mathrm{CPZ} 6+1)$ or 3-5 mice (for all other time points) from one representative of two independent experiments. The most relevant statistically significant differences after comparisons between groups are shown by two-way ANOVA with Bonferroni's test (Aii, Cii and E) and by Student's t-test (B and Dii) * $\mathrm{p} \leq$ $0.05, * \star p \leq 0.005, * \star * p \leq 0.001$. 

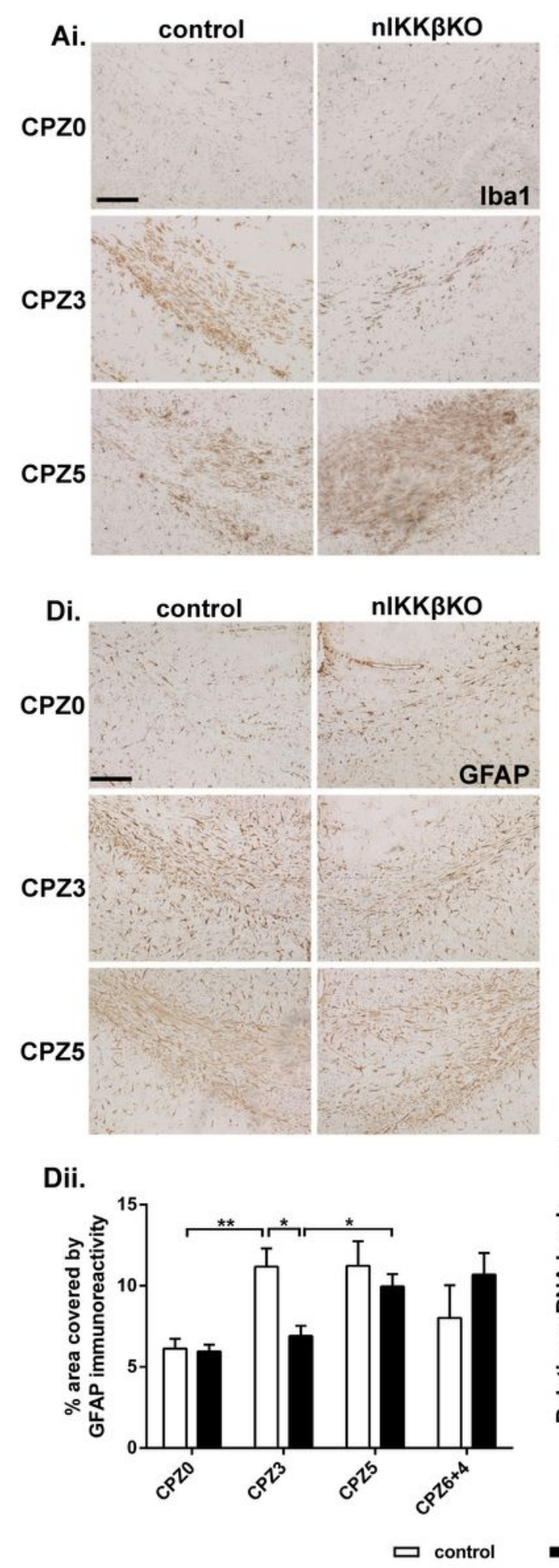
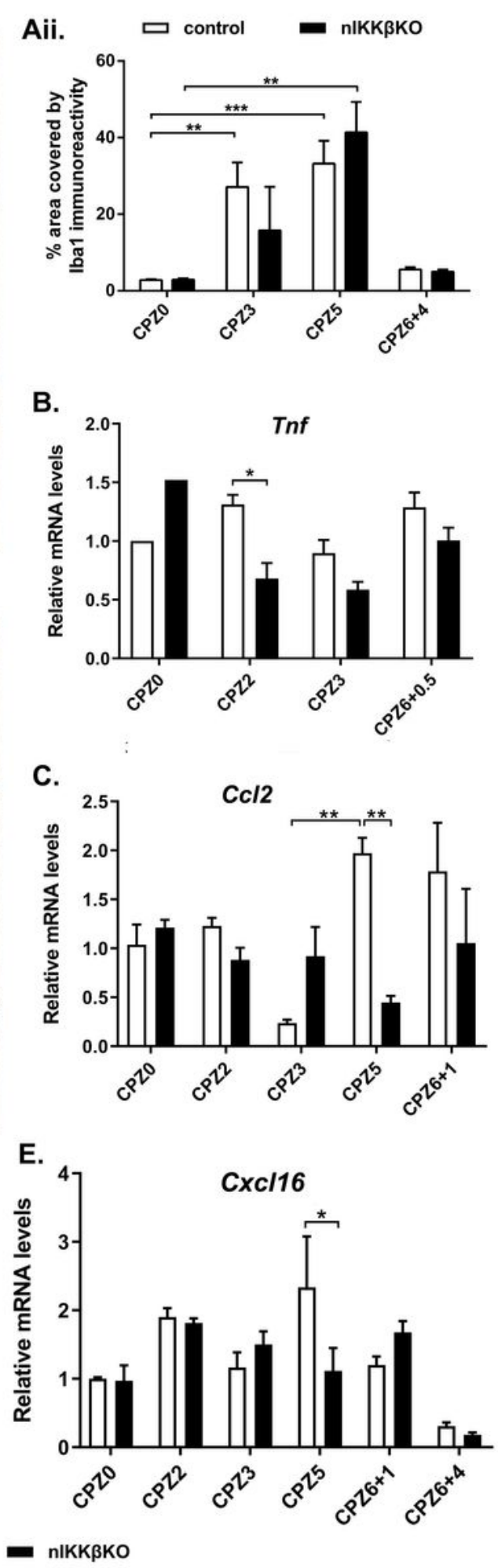

\section{Figure 5}

Neuronal IKK $\beta$ promotes astrocyte and microglia responses and inflammatory mediators in cuprizone demyelination. (Ai) Iba1 immunostaining of microglia and (Di) GFAP immunostaining of astrocytes in serial brain coronal paraffin sections from nIKK $\beta K O$ and control (IKKßff) naive (CPZO) or CPZ-fed CPZ3, CPZ5 and CPZ6+4 mice. Scale bars: $100 \mu \mathrm{M}$. Quantitative representation of areas covered by (Aii) lba1 immunoreactivity and (Dii) GFAP immunoreactivity in the corpus callosum in groups of nIKK $\beta K O$ and 
control mice represented in the photographs. (B, C and E) Differential expression of the inflammatory markers Tnf, $\mathrm{Ccl} 2$ and $\mathrm{Cxcl} 16$ relative to GusB in total mRNA isolates taken from nIKKßKO and IKKßff control brains from CPZ0 or CPZ-fed CPZ2, CPZ3, CPZ5, CPZ6+0.5, CPZ6+1 and CPZ6+4 mice. Results are means of 2 (nIKKßKO CPZ6+0.5, CPZ6+1, nIKKßKO CPZ6+4) or 3-5 mice (for all other groups and time points) from one representative of two independent experiments. The most relevant statistically significant differences after comparisons between groups are shown by Student's t-test (Aii and Dii) and by two-way ANOVA with Bonferroni's test $(\mathrm{B}, \mathrm{C}$ and $\mathrm{E})$. * $\mathrm{p} \leq 0.05$, $* * \mathrm{p} \leq 0.005$, ${ }^{* \star} \mathrm{p} \leq 0.001$.
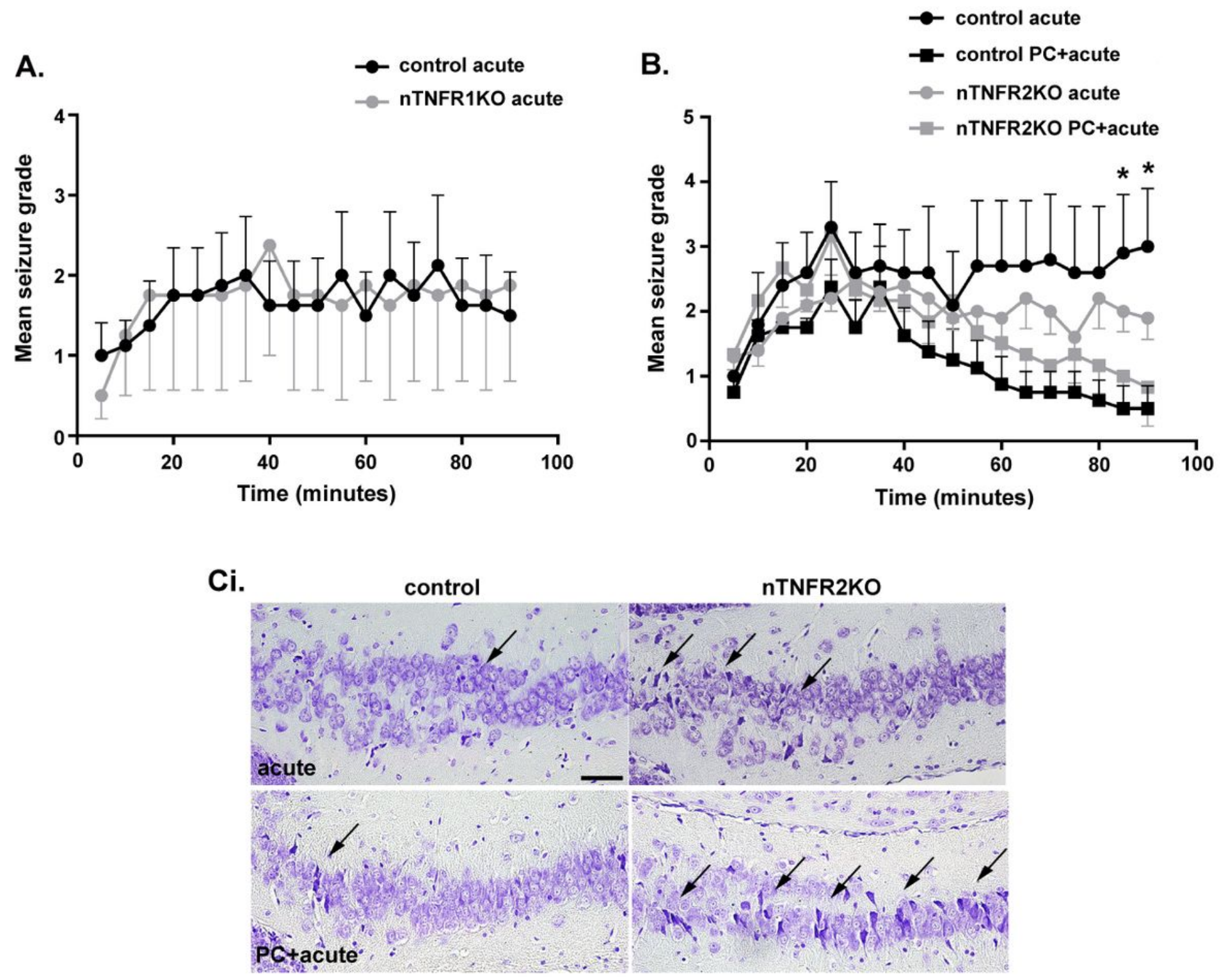

Figure 6

Preconditioning protection against kainic acid seizures and hippocampal neuron survival is reduced in nTNFR2KO mice. (A) Mean seizure scores recorded every 5 min after acute i.p. injection of $24 \mathrm{mg} / \mathrm{kg}$ kainic acid (KA) in nTNFR1KO ( $n=4$; grey circles) and TNFR1ff control ( $n=4$; black circles) mice. (B) Mean seizure scores recorded every 5 min after acute i.p. injection of $20 \mathrm{mg} / \mathrm{kg} \mathrm{KA}$ in nTNFR2KO $(\mathrm{n}=5$; grey circles) and TNFR2ff control ( $n=5$; black circles) mice, or after preconditioning with i.p injection of 15 
$\mathrm{mg} / \mathrm{kg} \mathrm{KA}$ followed after $24 \mathrm{~h}$ by i.p. injection of $20 \mathrm{mg} / \mathrm{kg} \mathrm{KA}$ in TNFR2KO ( $\mathrm{n}=4$; grey squares) and TNFR2ff control ( $n=4$; black squares) mice. (C) Cresyl violet (nissl) staining of brain coronal paraffin sections from mice shown in (B) taken 5 days after KA administration in non-preconditioned (acute) and KA preconditioned ( $\mathrm{PC}+$ acute) mice. Photographs showing pyknotic dead neurons in the CA3 region of the hippocampus (arrows). Scale bar: $50 \mu \mathrm{M}$. Results are representative of one or two (acute KA in nTNFR2KO and TNFR2ff control mice) independent experiments and are presented as mean values \pm SEM. Statistical significance after comparisons between acute KA versus preconditioning KA in each genotype are shown by one-way ANOVA with Bonferroni's test for each time point. * $p \leq 0.05$.
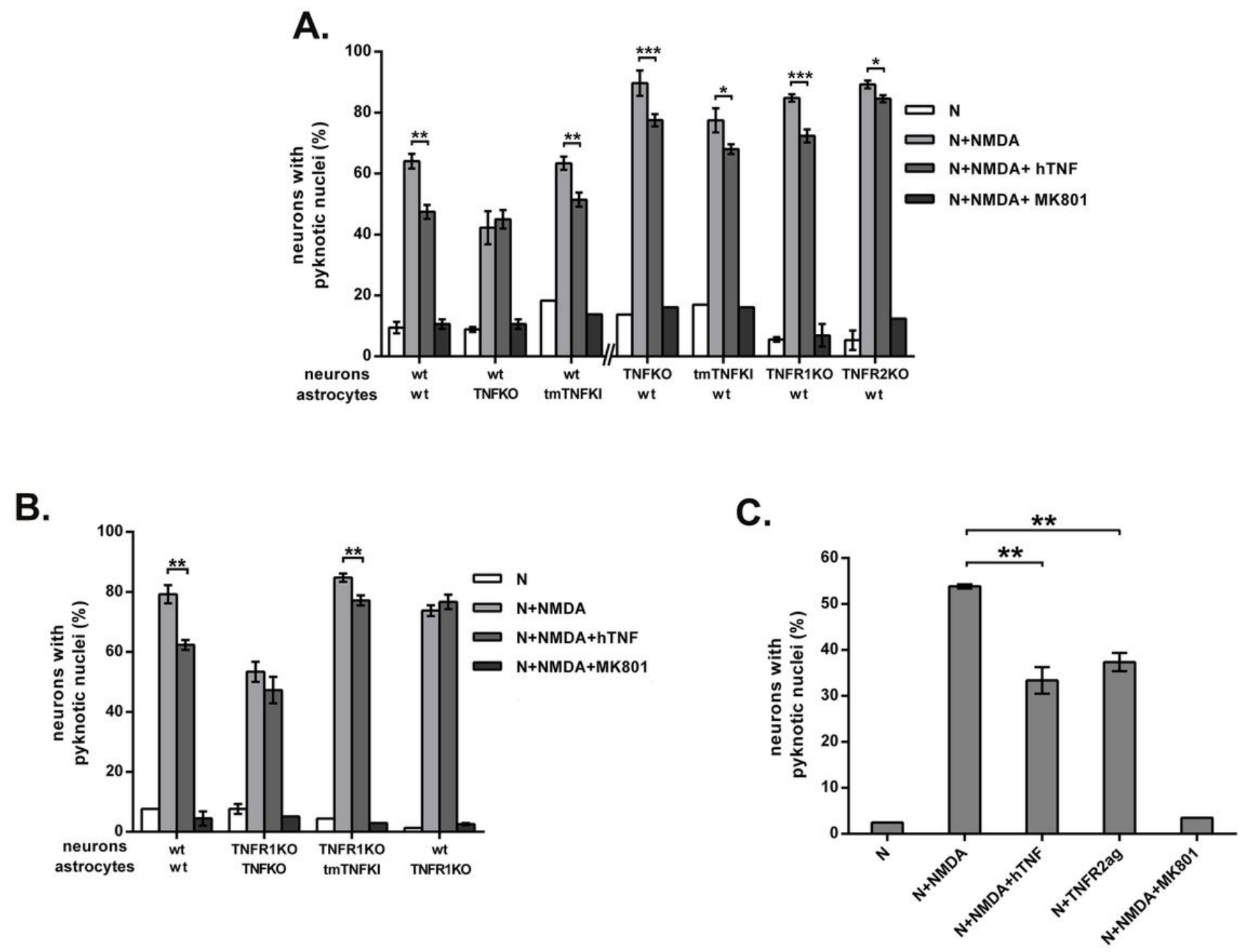

Figure 7

Astrocyte transmembrane TNF-neuronal TNFR2 interactions are necessary for TNF neuroprotection against NMDA excitotoxicity in vitro. (A and B) Addition of human TNF (hTNF) in astrocyte-neuron cocultures made from different combinations of WT, TNFKO and TNFRKO mice $24 \mathrm{~h}$ before NMDA-induced cell death. Specifically, neuron-astrocyte co-cultures at day in vitro 7 of co-culture (NA-DIV7) were pre- 
treated with $100 \mathrm{ng} / \mathrm{ml} \mathrm{hTNF}$ for $24 \mathrm{~h}$, NMDA excitotoxic death was induced with $50 \mu \mathrm{M} \mathrm{NMDA} / 10 \mu \mathrm{M}$ glycine on NA-DIV8, and death was measured after $22 \mathrm{~h}$. (C) Addition of a TNFR2 agonist in NA-DIV7 cocultures made from WT mice $24 \mathrm{~h}$ before NMDA-induced excitotoxicity provides equal neuroprotection against NMDA-induced cell death as hTNF. Results shown are means \pm SEM of duplicate or triplicate samples from one representative of two or three independent experiments. ${ }^{*} p \leq 0.05, * \star p \leq 0.005$, $* \star * p$ $\leq 0.001$, for pairwise comparisons between cells in each genotype combination by Student's t-test.

\section{Supplementary Files}

This is a list of supplementary files associated with this preprint. Click to download.

- SupplementaryFigure1.jpg

- SupplementaryFigure2.jpg

- SupplementaryFigure3.jpg

- SupplementaryFigure4.jpg 